\title{
THE COMPLEX NATURE OF THE INTERACTING SYSTEM NGC $7592^{1}$
}

\author{
Piero Rafanelli \\ Department of Astronomy, University of Padova, Vicolo Osservatorio 5, I-35122 Padova, Italy \\ Paolo Marziani \\ International School for Advanced Study, Viale Miramare 11, I-34014 Trieste, Italy \\ Received 22 May 1991; revised 25 September 1991
}

\begin{abstract}
In this paper we present a detailed analysis of the physical conditions and of the kinematical and dynamical properties of the system of interacting galaxies NGC 7592, the western component of which shows signs of Seyfert activity. Medium resolution long-slit spectra taken at different position angles along the nuclei show that the $\mathrm{H} \alpha, \mathrm{H} \beta$, [O III] $\lambda \lambda 4959,5007$, [N II] $\lambda \lambda 6548,6583$, [S II] $\lambda \lambda 6716,6731$, and [O II] $\lambda \lambda 3726,3729$ emission lines are spatially extended over a size comparable to the size of the system. The aspect and the kinematics of the galaxies suggest that a prograde encounter between the eastern and the western component of the system is occurring. The large $\mathrm{H} \alpha$ luminosity indicates that all galaxies are undergoing a strong burst of star formation, with a very large global star formation rate $\left(\approx 20 \mathscr{K} \odot \mathrm{yr}^{-1}\right)$. In the spectra of the nuclear and circumnuclear regions of the western component, the high ionization lines are redshifted by $\Delta v_{\mathrm{r}} \approx 50 \mathrm{~km} \mathrm{~s}^{-1}$ with respect to the low ionization lines, and their ratios indicate the simultaneous presence of thermally and nonthermally ionized gas. This is interpreted in terms of coexistence in the nucleus of the western component of Seyfert and Starburst activity, the last one being responsible for $\approx 75 \%$ of the $\mathrm{H} \alpha$ line luminosity. In the contact region between the eastern and the western component of NGC 7592, the presence of gas whose radial velocity increases approaching the Seyfert nucleus has been revealed. We suggest that this could be due to gas stripped from the eastern component, and which is infalling on the Seyfert nucleus.
\end{abstract}

\section{INTRODUCTION}

Gravitational interaction can enhance the star formation rate (SFR) in the disk and in the nuclear regions of galaxies, producing a starburst phenomenon in the most extreme cases, when the SFR cannot be sustained for a time comparable with the age of the galaxy (e.g., Weedman 1989). The link between the enhancement of SFR and interaction seems to be well established on a statistical basis, in the sense that interacting galaxies have larger emission line luminosity (Keel et al. 1985), stronger radio emission (Sulentic 1976; Stocke 1978; Heckman 1982; Hummel 1982) and higher farinfrared luminosity (Bushouse 1987; Lonsdale et al. 1984; Miley et al. 1985; Lawrence et al. 1989) than unperturbed and isolated galaxies. Moreover, the most powerful IRAS galaxies belong to interacting systems (Lawrence et al. 1989). It is less clear the role that interaction may have in inducing nonthermal, Seyfert type, nuclear activity. The works of Adams (1977), and Dahari (1984, 1985) suggested that there is an excess of morphologically perturbed and interacting systems among Seyfert galaxies in comparison with a sample of nonactive galaxies. Since the activity in Seyfert nuclei is believed to be powered by accretion onto a compact, massive object, it has often been suggested that gravitational instabilities produced by the interaction with a companion galaxy can lead the disk gas to move toward the nucleus of the galaxy (Simkin et al. 1980; Byrd et al. 1986), or even that the gas can be transferred from the companion to the active galaxy (e.g., Stockton 1982; de Robertis 1985). Connection between nuclear activity and gravitational interaction is not very firm on a statistical basis. Kennicutt \& Keel (1984), Keel et al. (1985), and Dahari (1985) have

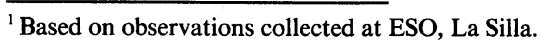

shown that interaction enhances both nonthermal activity and nuclear star formation, but the statistical significance of this effect for the nonthermal activity is very limited (Dahari \& de Robertis 1988b). It is noteworthy that more than $70 \%$ of the galaxies hosting an active nucleus do not show evident signs either of morphological peculiarities or interaction, since the percentage of interacting and morphologically peculiar objects is around, and probably less than $30 \%$. Furthermore, even the reality of the excess of interacting systems among Seyfert galaxies has been questioned by some recent works (Fuentes-Williams \& Stocke 1989). Gunn (1979) pointed out the difficulties that gas clouds may suffer in the dissipation of their angular momentum for reaching the galaxy nucleus, and in effectively providing the accreting matter for the massive compact object.

On the other side, starburst and nonthermal activity could be related phenomena. Emission line spectra of some low ionization objects suggest that they could be accounted for by a mixture of gas photoionized by both a thermal (as for $\mathrm{H}$ II regions) and a nonthermal contribution (as for active galactic nuclei; Keel et al. 1985; Rafanelli 1989). Furthermore, the presence of circumnuclear star formation has been revealed in NGC 1068 (see Wilson et al. 1988, for detailed reference concerning this object). Several evidences suggest that the far-infrared luminosity of Seyfert galaxies should be at least in part due to thermal reradiation of dust heated by hot, young O-B stars (e.g., Neugebauer et al. 1986; Rodriguez-Espinoza et al. 1987). Dahari \& de Robertis (1988a,b) in their comparative study of Starburst and Seyfert galaxies, pointed out that Starburst and Seyfert 2 galaxies have similar IRAS spectra, and similar $\mathrm{H} \alpha$ luminosity. However, starburst galaxies have narrower line widths than Seyfert 2 nuclei (Feldman et al. 1982; Balzano 1983), implying that the kinematics and dynamics of the emission line regions are 
probably different in the two classes (e.g., De Robertis \& Shaw 1988).

Weedman (1983) has proposed an evolutionary link between the starburst and Seyfert phenomenon. Since the gas velocity dispersion in the Starburst nuclei is probably too low for having equilibrium in the potential of the galactic bulge, remnants of star formation can collapse to a steady state configuration of radius $\approx 1 \mathrm{pc}$, and then accrete gas. Otherwise, mass loss during the later stages of the evolution of O-B stars could constitute a source of accreting matter for the central black hole (Norman \& Scoville 1988). Connections between the two phenomena have been barely exploited from the theoretical point of view, but also the observational evidence favoring a link remains until now sparse and circumstantial. If interaction is able to trigger a starburst phenomenon in an otherwise normal galaxy, the study of interacting systems with an ongoing starburst can provide constraints upon the geometry and dynamics of the encounter which are able at best to favor the occurrence and/or to strengthen the Starburst phenomenon. Intermediate objects, in which both a nuclear starburst and a Seyfert nucleus may simultaneously coexist, are laboratories in which the proposed scenarios connecting the two phenomena can be tested.

In this paper we present a detailed analysis of the physical conditions and of the kinematical and dynamical properties of NGC 7592. NGC 7592 $\equiv$ VV 731 is a system of close interacting galaxies. It is described in the 2 nd Reference Catalogue of Bright Galaxies (de Vaucouleurs \& de Vaucouleurs 1964 ) as a colliding pair of galaxies and by Vorontsov-Velyaminov (1979) as a pair of coalescents, whose nuclei are nearly aligned along the east-west direction. Markarian \& Lipovetskii (1977) identified the eastern component of the system as Mkn 928. Arkhipova et al. (1981) suggested that the system actually comprises three components in a common envelope, due to the presence of a third, smaller galactic body in the south. Arkhipova et al. (1981) noted that all nuclei of the three components show strong emission lines, and that the spectrum of the western nucleus is of Seyfert-type, with a broad component in $\mathrm{H} \alpha$ whose full width half maximum (FWHM) appeared to be $\gtrsim 1000 \mathrm{~km} \mathrm{~s}^{-1} \mathrm{FWHM}$. Further observations by Dahari (1985) did not confirm the presence of the broad component in $\mathrm{H} \alpha$, suggesting the classification of the western nucleus as a Seyfert 2. Dahari \& de Robertis (1988b) classified the same nucleus as intermediate between a Seyfert 2 and a starburst nucleus. In addition, NGC 7592 is a strong $I R A S$ source, whose far-infrared flux is dominated by thermal emission (Bushouse 1987).

\section{OBSERVATIONS AND DATA REDUCTION}

Images of NGC 7592 were taken on 11 November 1988 at the Cassegrain focus of the $1.82 \mathrm{~m}, f / 9$ telescope of the Asiago Observatory. The atmospheric seeing was typically $\approx 1.0^{\prime \prime}$ (FWHM). The Thompson CCD (pixel size 0.29") offered a field of view $\approx 2 \operatorname{arcmin} \times 3$ arcmin. Long slit spectra of NGC 7592 were obtained from 26 September to 29 September 1989, using the Boller and Chivens spectrograph on the ESO $1.52 \mathrm{~m}$ telescope. The detector was a high resolution RCA CCD (scale along the slit $0.68^{\prime \prime} / \mathrm{pixel}$ ), and was used for four settings with combined coverage 3350-7380 $\AA$. The slit was opened to a width of $\approx 2$ ". Atmospheric seeing was estimated to be $\approx 1.0^{\prime \prime}-1.5^{\prime \prime}$ (FWHM) during all the four nights of observation. On 26 September 1989 we used grating ESO No. 22 ruled at 1200 lines $\mathrm{mm}^{-1}$, while on 27
September, we used grating ESO No. 19 at 2nd order. Both settings gave a dispersion of $67 \AA / \mathrm{mm}$, and a mean spectral resolution of $2 \AA$ (FWHM). The spectral ranges covered were $6400-7400 \AA$ on 26 September, and 4650-5550 $\AA$ on 27 September. The spectrograph slit was oriented at P.A. $=100^{\circ}$ in order to intersect the eastern and the western nucleus of the system. On 28 September and 29 September we used grating ESO No. 27 ruled at 600 lines $\mathrm{mm}^{-1}$ giving a dispersion of $129 \AA / \mathrm{mm}$ and a mean spectral resolution of $4 \AA$. The covered spectral ranges were $5440-7380 \AA$ on 28 September and 3550-5450 $\AA$ on 29 September. The slit was oriented at P.A. $=32^{\circ}$ and $100^{\circ}$ during both nights. Due to guiding errors, the spectrum taken on 29 September at P.A. $=100^{\circ}$ is of poor quality. Its use has been limited to an estimate of the fluxes of the strongest lines of the nuclear spectra.

The reduction of the data was performed using the VISTA program, developed at Lick Observatory (Terndrup et al. 1984). Bias levels were subtracted from all frames, which were then divided by flatfield. No photometric calibration was applied to the images. The spectra were wavelength calibrated fitting third order polynomials to unblended emission lines in He-Ar comparison spectra recorded after each observation. rms was always less than $0.1 \AA$. The spectra were then sky subtracted and extinction corrected. The spectral response of the detector was measured and the spectra were placed to an absolute flux scale using observations of standard stars from the lists of Stone (1977), performed each night after the observation of NGC 7592.

Fluxes and positions of the lines were measured using the program RETICENT (Pritchet et al. 1981). Line centroids were evaluated weighing each wavelength bin with a weight $\propto I^{3}$ over a range of width \pm 1 FWHM, measured from the line center. The observed FWHM were corrected for instrumental width applying the relation (FWHM) ${ }^{2}$ $=(\mathrm{FWHM})_{\mathrm{obs}}^{2}-(\mathrm{FWHM})_{\text {instr }}^{2}$. The corrected line widths are reliable to $\pm 20 \%$.

\section{RESULTS}

\subsection{Morphology}

The following analysis of the morphology of the NGC 7592 system is based on the $R$ image, which is shown in Fig. 1 . The $V$ image is not presented here, because it is very similar to the $R$ one, although a little less deep. For the sake of clarity, we will refer to the western galactic body as to NGC $7592 \mathrm{~W}$, while NGC $7592 \mathrm{~A}$ will indicate its nucleus and the corresponding emitting region, identified on the spectra (see Table 1, Sec. 3.2). Similarly, NGC 7592 E and NGC 7592 S will indicate the eastern and the southern component, while their nuclei will be called NGC 7592 B and NGC 7592 C.

The nucleus $\mathrm{A}$ has a starlike appearance and it is surrounded by a nearly spheroidal envelope, with an extension in the west. This extension bends toward the north joining a bright and large wing, which resembles a spiral arm or perhaps a tidal feature. A faint extension, similar to a spiral arm, is visible in the south. The envelope around $\mathrm{A}$ is connected to a second galactic body, NGC $7592 \mathrm{E}$, whose nucleus (B), identified by Markarian \& Lipovetskii (1976) as the nucleus of $\mathrm{Mkn} 928$, is located at a distance $d_{\mathrm{AB}} \sim 11 \operatorname{arcsec}\left(\sim 4 h^{-1}\right.$ $\mathrm{kpc}$ ) from A at P.A. $=100^{\circ}$. The morphology of this component is peculiar. In the central region, $B$ is linked to two knots which extend up to $d " \sim 4$ arcsec to the east. In addition, a smooth extension is detected along the direction 
$\left(\mathrm{P} . \mathrm{A} .=40^{\circ}\right)$ joining $\mathrm{B}$ and C. An arc of condensations on the eastern side of NGC $7592 \mathrm{E}$ seems to be distorted in the direction of the nucleus of NGC $7592 \mathrm{~S}$, located at $d_{\mathrm{BO}} \approx 12.1 \operatorname{arcsec}\left(\approx 4.1 h^{-1} \mathrm{kpc}\right)$ from B at P.A. $=212^{\circ} . \mathrm{A}$ faint plume extends from the west side of NGC $7592 \mathrm{~S}$, bending toward south.

\subsection{Emission Line Regions}

The $\mathrm{H} \alpha, \mathrm{H} \beta$, [O III] $\lambda \lambda 4959,5007$, [N II] $\lambda \lambda 6548,6583$, [S II] $\lambda \lambda 6716,6731$, and [O II] $\lambda \lambda 3726,3729$ emission lines have been found to be spatially extended. Their overall extension is $\approx 30 \operatorname{arcsec} \approx 10.5 h^{-1} \mathrm{kpc}$ at P.A. $=100^{\circ}$ and $\approx 28$ arcsec $\approx 9.8 h^{-1} \mathrm{kpc}$ at P.A. $=212^{\circ}$. The size of the emitting regions is comparable with the size of the galaxies belonging to the system NGC 7592 as measured on their $R$ image. A careful analysis of the $\mathrm{H} \alpha$ profile along the slit permits to isolate six different emitting regions (A1,A,A2,B2,B,B1) along P.A. $=100^{\circ}$ and four regions $(\mathrm{C}, \mathrm{B} 4, \mathrm{~B}, \mathrm{~B} 3)$ along P.A. $=212^{\circ}$. The slit positions and the regions analyzed are marked in Fig. 1 [Plate 56]. A quantitative information on the location and the extension of the emitting regions is provided in Table 1 . Columns 1 and 2 list the slit P.A. at which each emitting region was observed and its identification; columns 3 and 4 quote their angular and projected linear size, while column 5 gives the identification of the nucleus (A or B) used as reference for the determination of the position angle and of the angular and projected linear distances quoted in columns 6-9. Column 10 reports the heliocentric radial velocities and their associated rms, derived measuring the following low-ionization lines: $\mathrm{H} \alpha$, $\mathrm{H} \beta$, [N II] $\lambda \lambda 6548,6583$, [S II] $\lambda \lambda 6716,6731$ and also [O II] $\lambda \lambda 3726,3729,\left[\mathrm{O}_{\mathrm{I}}\right] \lambda 6300$, and $\mathrm{H} \gamma$ for A, B, and C. The observed fluxes of the emission lines are quoted in Table 2. For lines which were present in more than one spectrum, the mean of the observed fluxes is given. Spectra of the nuclear regions NGC 7592 A, B, and C are shown in Figs. 2(a) and $2(\mathrm{~b})$.

The fluxes of the strongest emission lines are good to $\pm 15 \%$, whereas those below $2 \times 10^{-15} \mathrm{ergs} \mathrm{s}^{-1}$ can be uncertain by a factor of $\approx 2$. The Balmer lines of NGC $7592 \mathrm{~A}$ show features typical of a Seyfert 1.9 nucleus [Figs. 3(a) and 3(b) ]. Line fluxes for NGC $7592 \mathrm{~A}$ are in satisfactory agreement with the values reported by Dahari (1985), who had

TABLE 1. Emitting regions of the NGC 7592 system.

\begin{tabular}{|c|c|c|c|c|c|c|c|c|}
\hline \multirow[t]{2}{*}{ Sp. P.A. } & \multirow[t]{2}{*}{ Ident. } & \multicolumn{2}{|c|}{ Extension } & \multicolumn{4}{|c|}{ Distance } & \multirow{2}{*}{$\begin{array}{c}v_{\mathrm{r}} \\
\mathrm{km} / \mathrm{s}\end{array}$} \\
\hline & & arcsec & $h^{-1} p c$ & from & P.A. & arcsec & $h^{-1} p c$ & \\
\hline $100^{\circ}$ & A1 & 7.5 & 2700 & A & $280^{\circ}$ & 5.8 & 2030 & $7362 \pm 10$ \\
\hline$"$ & A & 3.4 & 1200 & A & - & 0.0 & 0 & $7330^{*} \pm 7$ \\
\hline$"$ & A2 & 3.4 & 1200 & A & $100^{\circ}$ & -3.4 & -1200 & $7330^{*} \pm 10$ \\
\hline$"$ & B2 & 4.1 & 1440 & B & $280^{\circ}$ & 4.1 & 1440 & $7300 \pm 10$ \\
\hline$"$ & B & 4.1 & 1440 & B & - & 0.0 & 0 & $7320 \pm 6$ \\
\hline$"$ & B1 & 6.8 & 2500 & B & $.100^{\circ}$ & -5.4 & -1900 & $7450 \pm 10$ \\
\hline $32^{\circ}$ & B3 & 7.4 & 2630 & B & $32^{\circ}$ & 5.8 & 2020 & $7375 \pm 10$ \\
\hline$n$ & B & 4.1 & 1430 & B & - & 0.0 & 0 & $7322 \pm 10$ \\
\hline$"$ & B4 & 4.1 & 1430 . & B & $212^{\circ}$ & -4.1 & -1430 & $7285 \pm 10$ \\
\hline$"$ & C & 11.6 & 40.60 & B & $212^{\circ}$ & -9.9 & -3450 & $7225 \pm 8$ \\
\hline
\end{tabular}

"see text classified this object as a Seyfert 2. The line fluxes of NGC $7592 \mathrm{~B}$ are reported in Table 2 both for P.A. $=100^{\circ}$ and P.A. $=32^{\circ}$. They differ by $\approx 10 \%-15 \%$, since at P.A. $=100^{\circ}$ the profile along the slit shows a contamination produced by the knot on its eastern side, see Fig. 1. The flux of $\mathrm{H} \alpha$ had been already measured by Bushouse (1987), who obtained a value just inbetween our results. Other line fluxes for NGC $7592 \mathrm{~B}$ and C are not available to our knowledge in literature. Extranuclear emitting regions located very close to the nuclei (as NGC 7592 A2 and B2) have continuum and line flux levels strongly sensitive to the variations of seeing, since they can be contaminated by the strong nuclear sources. We guess that the absolute flux calibration for these regions (A2 and B2) is accurate to within $\pm 30 \%$ uncertainty. The diagnostic diagrams proposed by Veilleux \& Osterbrock (1987) were used to analyze whether the ionization of the emitting regions in NGC 7592 is produced either by thermal sources ( $\mathrm{H}$ II regions) or by nonthermal sources, as it is the case for active galactic nuclei (AGN), Figs. 4(a)4(c). NGC 7592 A and A2 result to be ionized by a nonthermal source. All the other emitting regions can be classified as $\mathrm{H}$ II regions, with the exception of $\mathrm{A} 1$ for which a contribution to the ionization from NGC 7592 A cannot be excluded [see Fig. 4(b) ]. This classification has been also tested with the diagnostic diagrams based on the line ratios [O II] $\lambda \lambda 3726,3729 /[\mathrm{O} \mathrm{III}] \lambda$ 5007, [O III] $\lambda$ 5007/H $\beta$ and [N II] $\lambda 6583 / \mathrm{H} \alpha$ (Baldwin et al. 1981), which further allow to identify gas ionized by shock heating. It is confirmed that $B$, B3, B4, and C are typical $\mathrm{H}$ II regions, while A can be classified either as a power-law galaxy or as a shock-heated galaxy.

Values of electron density, Balmer decrement and internal extinction for each emitting region are quoted in Table 3 . The electron density $n_{\mathrm{e}}$ has been estimated from the ratio [S II] $\lambda 6716 /[$ S II] $\lambda 6731$, assuming an electron temperature $T_{\mathrm{e}} \approx 10000 \mathrm{~K}$. The observation of a standard star right after each exposure of NGC 7592 allowed to remove with good accuracy the effect of the Fraunhofer B absorption band. Uncertainty in the density estimates is approximately a factor 2 , for density values $\gtrsim 100 \mathrm{~cm}^{-3}$. Balmer decrements have been computed after correction for galactic extinction, being $\mathrm{E}(B-V)_{\mathrm{gal}}=0.015$ (Burstein 1986). The internal reddening $c$ and the corresponding $\mathrm{E}(B-V)$ has been computed using the Whitford reddening curve as parameterized by Miller \& Mathews (1972). An intrinsic ratio $\mathrm{H} \alpha / \mathrm{H} \beta \approx 2.85$ has been adopted for regions photoionized by thermal sources, while for regions whose ionization source is nonthermal, it has been assumed a ratio $\mathrm{H} \alpha / \mathrm{H} \beta=3.1$ (Ferland \& Netzer 1983).

Regions located in the outer parts of the galaxies NGC $7592 \mathrm{E}$ and $\mathrm{W}$ (B1, B3 and B4, and A1) are characterized by very low electron density, $n_{\mathrm{e}} \sim 10 \mathrm{~cm}^{-3}$, and by narrow emission lines FWHM $\sim 140 \mathrm{~km} \mathrm{~s}^{-1}$. The properties of the nuclear regions $\mathrm{A}, \mathrm{B}$, and $\mathrm{C}$ and of the regions between $\mathrm{B}$ and A, B2 and A2, are discussed more extensively in the following subsections.

NGC $7592 A$. Figures 3a and 3b display the high resolution spectra of NGC $7592 \mathrm{~A}$, which were used to measure the emission line widths and to analyze the line profiles. Most lines are asymmetric toward the red. The asymmetry is due to a semibroad component displaced to the red, which is present in the [O III] $\lambda \lambda 4959,5007$, [O I] $\lambda 6300$, but not in $\mathrm{H} \beta$ and in the [S II] $\lambda \lambda 6716,6731$ lines. The FWZI of the semibroad component, measured on the [O III] $\lambda 5007$ line is $\approx 1800 \mathrm{~km} \mathrm{~s}^{-1}$, while the FWZI of $\mathrm{H} \beta$ is $\approx 850 \mathrm{~km} \mathrm{~s}^{-1}$. A 
TABLE 2. Emission line fluxes. ${ }^{1}$

\begin{tabular}{|c|c|c|c|c|c|c|c|c|c|c|c|}
\hline \multicolumn{2}{|c|}{ Line Id. } & \multirow{3}{*}{$\begin{array}{l}\text { A } \\
11.7\end{array}$} & \multirow{3}{*}{$\begin{array}{r}\mathrm{A} 1 \\
5.9\end{array}$} & \multirow{3}{*}{$\begin{array}{r}\mathrm{A} 2 \\
1.7\end{array}$} & \multicolumn{2}{|c|}{ B } & \multirow{3}{*}{$\begin{array}{r}\text { B1 } \\
\mathbf{8 . 3}\end{array}$} & \multirow{3}{*}{$\begin{array}{r}\text { B2 } \\
3.3\end{array}$} & \multirow{3}{*}{$\begin{array}{r}\text { B3 } \\
4.8\end{array}$} & \multirow{3}{*}{$\begin{array}{r}\text { B4 } \\
4.0\end{array}$} & \multirow{3}{*}{$\begin{array}{l}\text { C } \\
6.0\end{array}$} \\
\hline & & & & & \multirow{2}{*}{$\begin{array}{r}P . A .=100^{\circ} \\
12.0\end{array}$} & \multirow{2}{*}{$\begin{array}{r}P . A .=32^{\circ} \\
10.3\end{array}$} & & & & & \\
\hline$[S I I]$ & $\lambda 6731$ & & & & & & & & & & \\
\hline$[S I I]$ & $\lambda 6716$ & 14.8 & 9.0 & 2.0 & 15.6 & 13.0 & 11.6 & 4.5 & 7.2 & 5.0 & 7.3 \\
\hline$[N I I]$ & $\lambda 6583$ & 32.2 & 16.8 & 6.2 & 27.5 & 22.9 & 18.1 & 10.7 & 13.8 & 8.4 & 14.7 \\
\hline $\mathrm{H} \alpha(\mathrm{NC})$ & $\lambda 6563$ & 44.7 & 45.5 & 13.2 & 80.6 & 69.7 & 54.5 & 35.3 & 37.6 & 25.8 & 61.1 \\
\hline $\mathrm{H} \alpha(\mathrm{BC})$ & $\lambda 6563$ & 31.2 & - & - & - & - & - & - & - & - & - \\
\hline$[N I I]$ & $\lambda 6548$ & 10.7 & 6.3 & 2.6 & 9.3 & 7.6 & 6.0 & 3.5 & 4.6 & 2.6 & 5.6 \\
\hline$[O I]$ & $\lambda 6300$ & 6.1 & $<3$ & 1.5 & 2.5 & 1.5 & 1.5 & 0.5 & 2.5 & $\sim 0.5$ & 2.8 \\
\hline HeI & $\lambda 5876$ & 2.3 & - & - & $<3$ & - & - & - & - & - & $<3$ \\
\hline$[N I]$ & $\lambda 5198$ & 1.5 & - & - & - & - & - & - & - & - & - \\
\hline$[O I I I]$ & $\lambda 5007$ & 32.2 & 7.9 & 8.3 & 12.9 & 11.0 & 9.9 & 7.2 & 5.6 & 4.5 & 15.4 \\
\hline$[O I I I]$ & $\lambda 4959$ & 11.2 & 2.7 & 2.8 & 4.3 & 4.0 & 3.4 & 2.6 & 2.2 & 1.5 & 2.2 \\
\hline $\mathrm{H} \beta$ & $\lambda 4861$ & 11.7 & 9.8 & 3.2 & 17.0 & 13.7 & 15.3 & 10.1 & 8.6 & 4.9 & 14.8 \\
\hline $\mathrm{H} \gamma$ & $\lambda 4340$ & 4.6 & n.a. & n.a. & 6.0 & 4.6 & n.a. & n.a. & 4.4 & - & 5.6 \\
\hline$[O I I]$ & $\lambda 3727$ & 30.4 & n.a. & n.a. & 30.3 & 25.6 & n.a. & n.a. & 15.4 & 6.8 & 26.2 \\
\hline
\end{tabular}

-: not detected

n.a.: not available.

${ }^{1}$ Line fluxes are in units of $10^{-15} \mathrm{ergs} \mathrm{cm}^{-2} \mathrm{~s}^{-1}$

TABLE 2(a). NGC 7592A. Fluxes of line components.*

\begin{tabular}{lcccc}
\hline \hline \multicolumn{1}{c}{ Line Id. } & Blue & Red & Semibroad \\
& & & & \\
\hline & & & & \\
{$[S I I]$} & $\lambda 6731$ & 5.3 & 7.3 & - \\
{$[S I I]$} & $\lambda 6716$ & 6.3 & 7.3 & - \\
{$[N I I]$} & $\lambda 6583$ & 17 & 8.7 & 13.5 \\
$\mathrm{H} \alpha$ & $\lambda 6563$ & 31.5 & 11.4 & $22.4^{(\circ)}$ \\
{$[N I I]$} & $\lambda 6548$ & $5.7^{(\dagger)}$ & $2.9^{(\dagger)}$ & $4.8^{(\dagger)}$ \\
{$[O I]$} & $\lambda 6300$ & $\sim 2.4$ & $\sim 2.4$ & $\sim 2.0$ \\
{$[O I I I]$} & $\lambda 5007$ & 11.4 & 9.5 & 12.3 \\
{$[O I I I]$} & $\lambda 4959$ & 3.8 & 3.2 & 4.1 \\
$\mathrm{H} \beta$ & $\lambda 4861$ & 7.8 & 3.9 & 2.4 \\
& & & & \\
\hline
\end{tabular}

(*) Line fluxes are in units of $10^{-15} \mathrm{ergs} \mathrm{cm}^{-2} \mathrm{~s}^{-1}$.

(०)Estimated as the difference between the total flux of the blend

$\mathrm{H} \alpha+[\mathrm{N}$ II $]$ and the sum of all other lines.

${ }^{(\dagger)}$ Assumed equal to $1 / 3 I([\mathrm{~N}$ II $] \lambda 6584)$.

faint broad component is present below the system of lines [N II] $\lambda \lambda 6548,6583$ and $\mathrm{H} \alpha$. Fluxes were estimated employing a multicomponent fit with four Gaussian curves modeling the narrow and broad components of $\mathrm{H} \alpha$ ( $\mathrm{NC}$ and BC) and the [ $\left.\mathrm{N}_{\text {II }}\right] \lambda \lambda 6548,6583$ profiles. Their values are reported in Table 2. However it should be remarked that a semibroad component below the $\mathrm{H} \alpha$ and [N II] $\lambda \lambda 6548,6583$ lines could produce an apparent $\mathrm{H} \alpha$ broad component. This seems to be the case, since the upper radial velocity of the broad component is consistent with that expected for the semibroad component of the [N II] $\lambda 6583$ line.

All emission lines $(\mathrm{H} \alpha, \mathrm{H} \beta,[\mathrm{S}$ II] $\lambda \lambda 6716,6731$, [N II] $\lambda \lambda 6548,6583$, [O I] $\lambda 6300$, [O III] $\lambda \lambda 4959,5007)$, show a double peaked core [see Figs. 5(a) and 5(b), where the line cores of the $\mathrm{H} \beta$ and [O $\mathrm{III}] \lambda 5007$ are reproduced; hereafter 

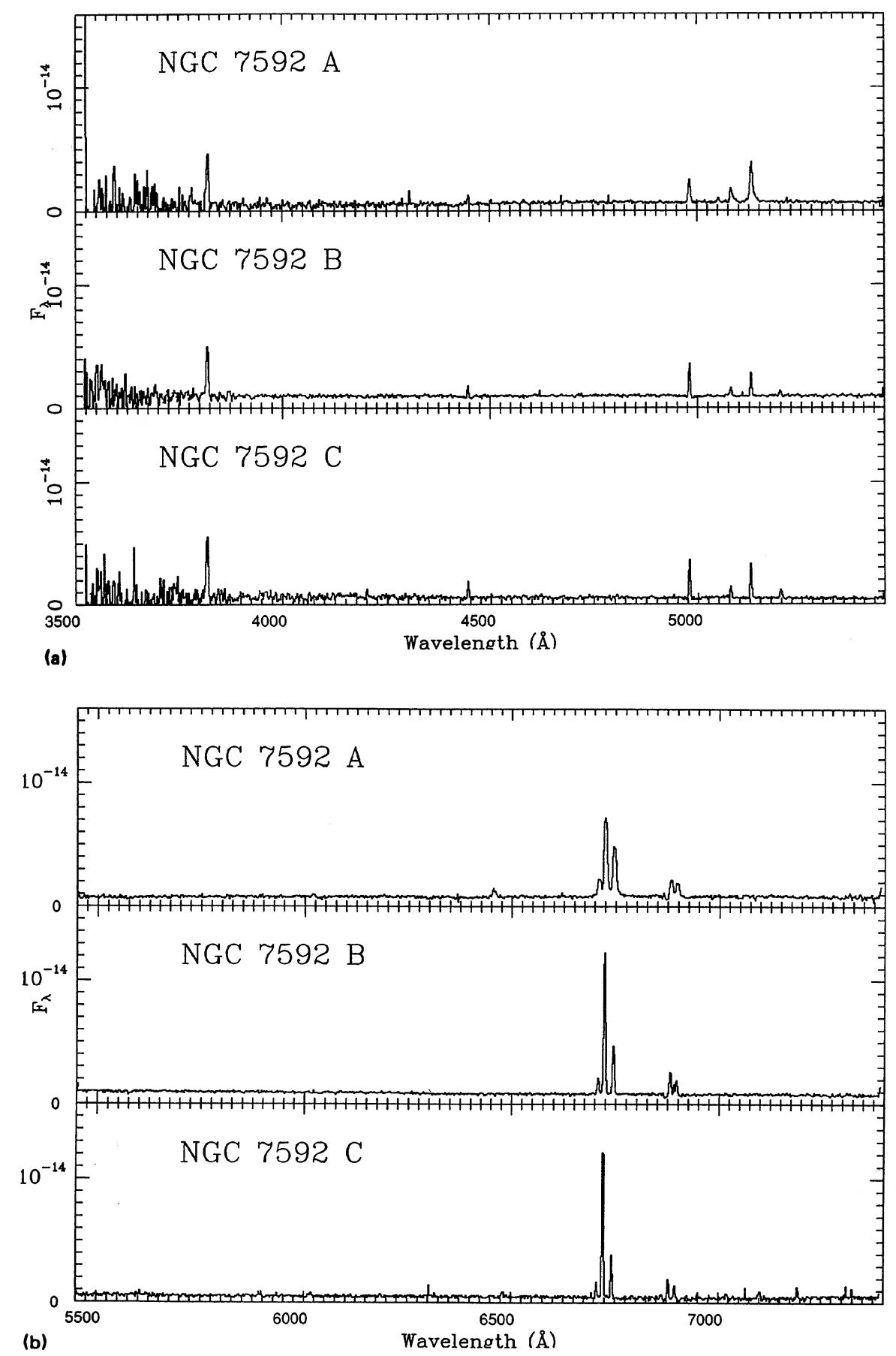

Fig. 2. (a) Blue and (b) red specra, respectively of NGC $7592 \mathrm{~A}, \mathrm{~B}$, and C. Vertical scale is flux per unit wavelength (ergs cm ${ }^{-2} \mathrm{~s}^{-1} \AA^{-1}$ ); horizontal scale is wavelength in $\AA$.

we will refer to the lower and higher radial velocity component as the blue and red component). The only possible exception is the [ $\left.\mathrm{N}_{\mathrm{I}}\right] \lambda 5198$ line, whose faintness does not allow any reliable consideration on its profile. The average heliocentric radial velocity of the blue component is $v_{r, B}=7280 \pm 7 \mathrm{~km} \mathrm{~s}^{-1}$ for all lines with the exception of [O III] $\lambda \lambda 4959,5007$, for which $v_{r, B}=7330 \pm 10 \mathrm{~km} \mathrm{~s}^{-1}$. The radial velocities of the red component of the [O III] $\lambda \lambda 4949,5007$ lines and the value averaged over all the other lines are $v_{r, R} \lesssim 7510 \mathrm{~km} \mathrm{~s}^{-1}$ and $v_{r, R}=7460 \pm 50 \mathrm{~km} \mathrm{~s}^{-1}$, respectively. The extension along the slit of the red component of $\mathrm{H} \beta$ and $\mathrm{H} \alpha$, and of the [N II] and [S II] lines [see Fig. 6(a) and the next section] suggests that this component corresponds to the red component seen in the [O III] lines. The radial velocity of the blue peak of $\mathrm{H} \beta$ is on the contrary significantly lower than that measured for the blue peak of 

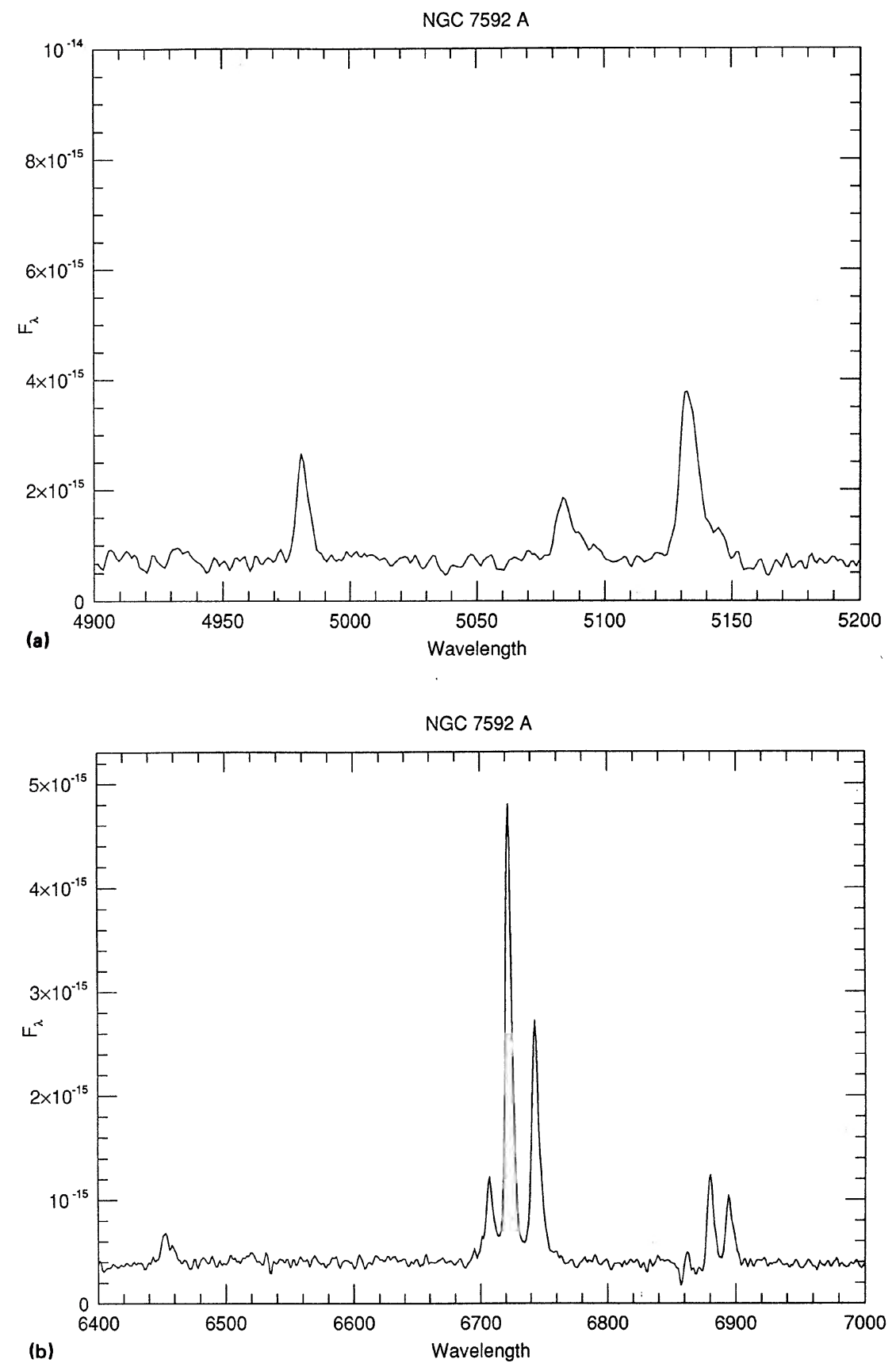

FIG. 3. High resolution spectra of NGC $7592 \mathrm{~A}$ : (a) profiles of $\mathrm{H} \beta$ and of the [O III] $\lambda \lambda$ 4959,5007 lines; (b) the $\mathrm{H} \alpha$ and $\left[\mathrm{N}_{\mathrm{II}}\right] \lambda \lambda 6548,6583$ blend. Units are as for Fig. 2.

the [O III] $\lambda 5007$ line. Although the difference is small, $\Delta v_{\mathrm{r}} \approx 50 \mathrm{~km} \mathrm{~s}^{-1}$, it is also confirmed by the radial velocity measurements on the extended emitting regions (see the next section).

The measurement of the FWHM of the double peaked emission lines is then affected by the relative strength of their two components. FWHM values of [O III] $\lambda \lambda 4959,5007$ and [O I] $\lambda 6300$ (490 and $280 \mathrm{~km} \mathrm{~s}^{-1}$, respectively) are larger than those of the other lines $\left(\approx 200-220 \mathrm{~km} \mathrm{~s}^{-1}\right)$. This happens because the red and blue components have a similar peak intensity in the [O III] $\lambda \lambda 4959,5007$ and [O I] $\lambda 6300$ lines, while the red component is fainter than the blue one $\left(I_{R} \lesssim 0.5 I_{B}\right)$ in $\mathrm{H} \alpha, \mathrm{H} \beta$, and [N II] $\lambda \lambda 6548,6583$. The FWHM of $\mathrm{H} \beta, \mathrm{H} \alpha$, and [N II] $\lambda \lambda 6548,6583$ should be therefore regarded as an estimate of the width of the blue component.

We have been able to separate the double-peaked profiles in the two core components and in the underlying semibroad component, and hence to report the line ratios of the core components separately in the diagnostic diagrams. The red

\section{(C) American Astronomical Society - Provided by the NASA Astrophysics Data System}



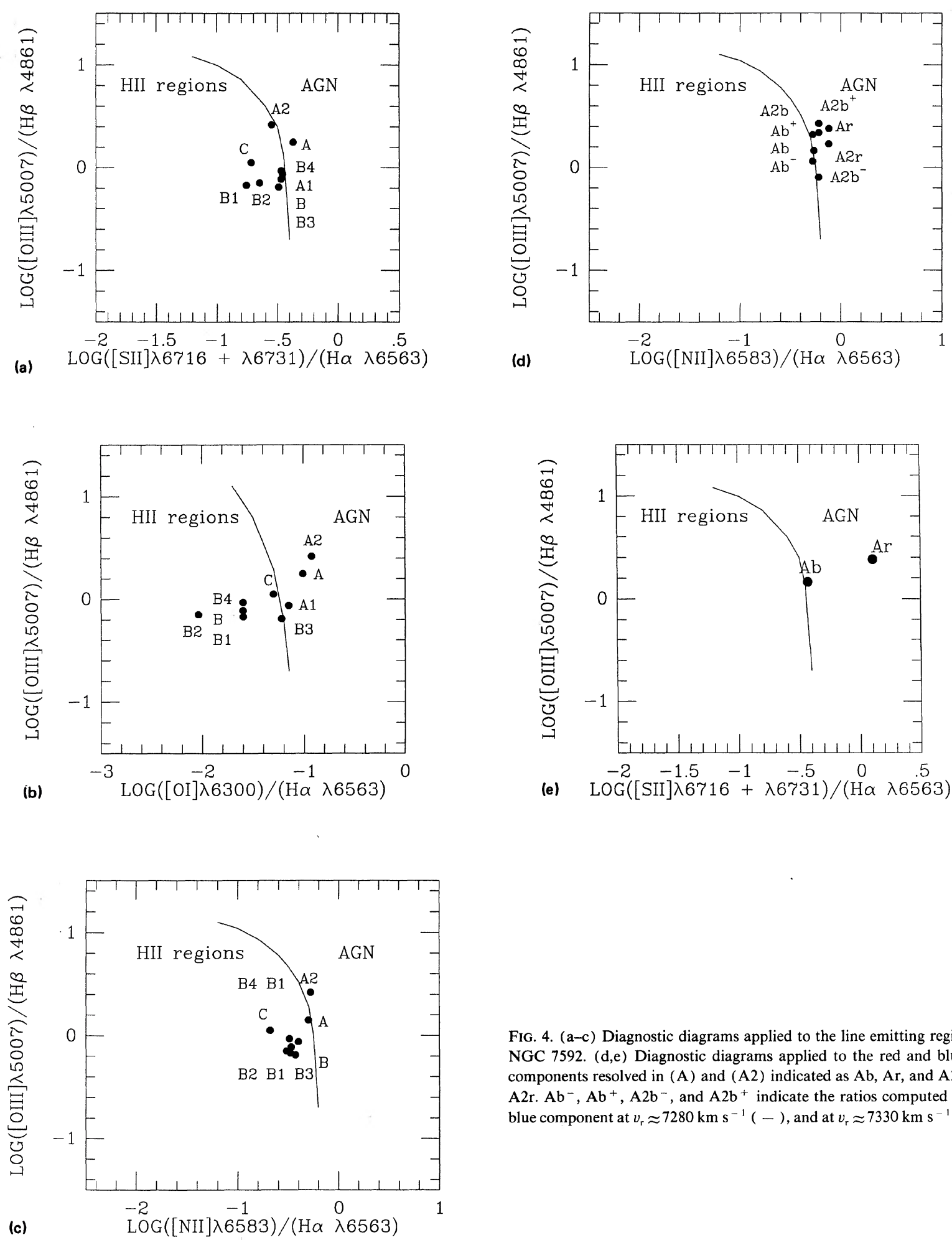

Fig. 4. (a-c) Diagnostic diagrams applied to the line emitting regions of NGC 7592. (d,e) Diagnostic diagrams applied to the red and blue line components resolved in (A) and (A2) indicated as $\mathrm{Ab}, \mathrm{Ar}$, and $\mathrm{A} 2 \mathrm{~b}$ and $\mathrm{A} 2 \mathrm{r} . \mathrm{Ab}^{-}, \mathrm{Ab}^{+}, \mathrm{A} 2 \mathrm{~b}^{-}$, and $\mathrm{A} 2 \mathrm{~b}^{+}$indicate the ratios computed on the blue component at $v_{\mathrm{r}} \approx 7280 \mathrm{~km} \mathrm{~s}^{-1}(-)$, and at $v_{\mathrm{r}} \approx 7330 \mathrm{~km} \mathrm{~s}^{-1}(+)$. 
TABLE 3. Main features of the emitting regions.

\begin{tabular}{|c|c|c|c|c|c|c|c|c|c|c|}
\hline & \multirow[t]{2}{*}{ A } & \multirow[t]{2}{*}{ A1 } & \multirow[t]{2}{*}{ A2 } & \multicolumn{2}{|c|}{ B } & \multirow[t]{2}{*}{ B1 } & \multirow[t]{2}{*}{ B2 } & \multirow[t]{2}{*}{ B3 } & \multirow[t]{2}{*}{ B4 } & \multirow[t]{2}{*}{$\mathrm{C}$} \\
\hline & & & & $P . A .=100^{\circ}$ & P.A. $=32^{\circ}$ & & & & & \\
\hline $\mathrm{n}_{e}\left(\mathrm{~cm}^{-3}\right)$ & 550 & $\lesssim 10$ & 180 & 150 & - & 40 & 70 & $\lesssim 10$ & 180 & 210 \\
\hline $\mathrm{H} \alpha / \mathrm{H} \beta / \mathrm{H} \gamma$ & $4.1 / 1.0 / 0.39$ & $5.5 / 1.0 /-$ & $4.4 / 1.0 /-$ & $5.1 / 1.0 / 0.37$ & $4.8 / 1.0 / 0.37$ & $3.8 / 1.0 /-$ & $3.8 / 1.0 /-$ & $4.8 / 1.0 /-$ & $5.8 / 1.0 /-$ & $4.9 / 1.0 / 0.38$ \\
\hline c & 0.37 & 0.87 & 0.46 & 0.77 & 0.69 & 0.38 & 0.38 & 0.71 & 0.94 & 0.72 \\
\hline $\mathrm{E}(\mathrm{B}-\mathrm{V})$ & 0.28 & 0.67 & 0.35 & 0.60 & 0.53 & 0.29 & 0.29 & 0.55 & 0.72 & 0.55 \\
\hline $\mathrm{L}_{c}(\mathrm{H} \alpha) \cdot h^{2}\left(\operatorname{erg~s}^{-1}\right)$ & $4.1 \times 10^{40}$ & $1.4 \times 10^{41}$ & $2.0 \times 10^{40}$ & $2.2 \times 10^{41}$ & $1.8 \times 10^{41}$ & $7.4 \times 10^{40}$ & $4.8 \times 10^{40}$ & $1.0 \times 10^{41}$ & $1.0 \times 10^{41}$ & $1.5 \times 10^{41}$ \\
\hline$h^{2}$.S.F.R $\left(\mathrm{M}_{\odot} y r^{-1}\right), \mathrm{M}>10 \mathrm{M}_{\odot}$ & 0.06 & 0.20 & 0.28 & 0.31 & 0.25 & 0.1 & 0.068 & 0.14 & 0.14 & 0.21 \\
\hline$h^{2}$.S.F.R. $\left(\mathrm{M}_{\odot} y r^{-1}\right)$, total & 0.36 & 1.25 & 0.18 & 1.96 & 1.6 & 0.66 & 0.43 & 0.89 & 0.89 & 1.33 \\
\hline Projected Area $\left(p c^{2} h^{2}\right)$ & $8.3 \times 10^{5}$ & $1.84 \times 10^{6}$ & $8.33 \times 10^{5}$ & $1.00 \times 10^{6}$ & $1.0 \times 10^{6}$ & $1.7 \times 10^{6}$ & $1.0 \times 10^{6}$ & $1.8 \times 10^{6}$ & $1.0 \times 10^{6}$ & $2.84 \times 10^{6}$ \\
\hline SFR/area $\left(\mathrm{M}_{\odot} y r^{-1} p c^{-2}\right)$ & $4.3 \times 10^{-7}$ & $6.8 \times 10^{-7}$ & $2.1 \times 10^{-7}$ & $2.0 \times 10^{-6}$ & $1.6 \times 10^{-6}$ & $3.9 \times 10^{-7}$ & $4.3 \times 10^{-7}$ & $4.9 \times 10^{-7}$ & $8.9 \times 10^{-7}$ & $4.7 \times 10^{-7}$ \\
\hline
\end{tabular}

component, indicated as Ar, is located among AGN in all the three diagnostic diagrams, while the blue one $(\mathrm{Ab})$ has a more ambiguous location [see Figs. 4(d) and 4(e)]. An estimate of the flux values for each component is reported in Table 2(a). The ionization degree of the red component ([O III ] $\lambda 5007 / \mathrm{H} \beta \approx 2.5$ ) is higher than that of the blue component ( $\left[\mathrm{O}_{\mathrm{III}}\right] \lambda 5007 / \mathrm{H} \beta \approx 1.5$ ). The electron density of the gas emitting the red component, $n_{\mathrm{e}} \gtrsim 600 \mathrm{~cm}^{-3}$, appears to be somewhat higher than that of the blue one, $n_{\mathrm{e}} \lesssim 250 \mathrm{~cm}^{-3}$.

The ambiguous location of $\mathrm{Ab}$ in the diagnostic diagrams may imply that the emitting gas is either partly ionized by the same nonthermal source ionizing Ar, or by an internal nonthermal source.

Support to the latter suggestion is provided by the radial velocity difference between the peaks of the blue components of [O III] $\lambda \lambda 4959,5007$ and $\mathrm{H} \beta$. This implies that the blue component of the Balmer and [O III] lines originates to some extent in different gaseous media, which are also in different kinematical conditions. If this is true, [O $\mathrm{III}]$ $\lambda 5007$ and $\mathrm{H} \beta$ are due to a mixture of contributions: the

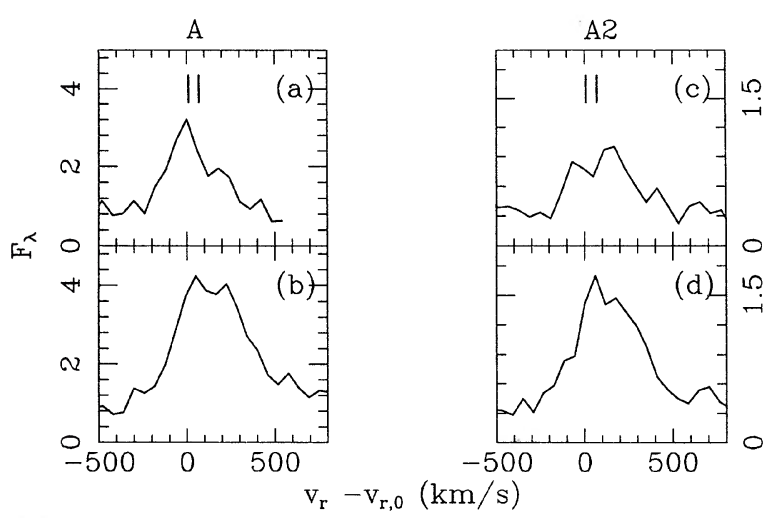

Fig. 5. Comparison of the $\mathrm{H} \beta$ and [O III] $\lambda 5007$ line profiles: (a) and (b) are for NGC $7592 \mathrm{~A}$; (c) and (d) for region NGC 7592 A2. Vertical scale is flux per unit wavelength (ergs s $\mathrm{s}^{-1} \mathrm{~cm}^{-2} \AA^{-1}$ ); horizontal scale is radial velocity difference from the peak radial velocity of $\mathrm{H} \beta, \approx 7270 \mathrm{~km} \mathrm{~s}^{-1}$. The two thick marks indicate $\Delta v_{\mathrm{r}}=0 \mathrm{~km} \mathrm{~s}^{-1}$ and $\Delta v_{\mathrm{r}}=60 \mathrm{~km} \mathrm{~s}^{-1}$. ratio of the intensities of the [O III] $\lambda 5007$ line and $\mathrm{H} \beta$ at a wavelength corresponding to $v_{\mathrm{r}} \approx 7280 \mathrm{~km} \mathrm{~s}^{-1}$, namely the peak velocity of the blue component of all lines but [O III], is $\lesssim 1$, indicating the presence of low ionization emitting gas in the blue component. The same intensity ratio at $v_{\mathrm{r}} \approx 7330$ $\mathrm{km} \mathrm{s}^{-1}$, is $>1$, indicating the presence of highly ionized gas too. These results are confirmed by the $[\mathrm{O} \mathrm{III]} \lambda 5007 / \mathrm{H} \beta$ intensity ratios at $v_{\mathrm{r}} \approx 7280 \mathrm{~km} \mathrm{~s}^{-1}$ and $v_{\mathrm{r}} \approx 7330 \mathrm{~km} \mathrm{~s}^{-1}$ measured in the spectrum of the close extranuclear region A2 (see further details in the next subsection). The $\mathrm{H} \alpha$ broad component can be accounted for by the blend of the semibroad components emitted by the [N II] $\lambda \lambda 6548,6583$ doublet, and perhaps $\mathrm{H} \alpha$. The upper radial velocity at which the broad feature is detected is consistent with the upper radial velocity of the [O III] $\lambda 5007$ semibroad component.

Although it is possible to estimate the contribution of the semibroad component in the [N $\mathrm{NI}] \lambda 6583$ profile, it is not possible to use directly the diagnostic diagrams of Veilleux and Osterbrock, since the estimate of its flux contribution in $\mathrm{H} \alpha$ is too uncertain. We thus employed the ratio [O III] $\lambda 5007 / \mathrm{H} \beta$ and the ratio [O I ] $\lambda$ 6300/[N II] $\lambda$ 6583. A comparison with the values provided by Veilleux \& Osterbrock (1987) suggests that the line ratios found for the semibroad component are rather typical of regions ionized by nonthermal sources.

The emission regions between $A$ and $B$. The spectrum of region $\mathrm{A} 2$ shows emission line profiles similar to those seen in the spectrum of the Seyfert nucleus, NGC 7592 A, but without the semibroad component. The [O III] $\lambda 5007$ and $\mathrm{H} \beta$ profiles are given in Figs. 5(c) and 5(d). The line cores are double peaked, and the radial velocity of the blue component of [O III] $\lambda 5007$ is in this case higher than that of $\mathrm{H} \beta$ by $\Delta v_{\mathrm{r}} \approx 100 \mathrm{~km} \mathrm{~s}^{-1}$. Unlike NGC $7592 \mathrm{~A}$, the ionization degree in NGC $7592 \mathrm{~A} 2$ is higher in the blue component ([O III $] \lambda 5007 / \mathrm{H} \beta \approx 2.5$ than in the red one ([O III] $\lambda 5007 / \mathrm{H} \beta \approx 1.5)$. In the diagnostic diagram of Fig. $5(\mathrm{~d})$ both components (labeled as A2b and A2r) are located among the nonthermally ionized regions.

The $\mathrm{H} \beta$ profile displays a minimum between the blue and the red component at the wavelength corresponding to the radial velocity $v_{\mathrm{r}} \approx 7320 \mathrm{~km} \mathrm{~s}^{-1}$, namely the peak velocity of the blue component of [O III] $\lambda$ 5007. The [O III] $\lambda 5007 / \mathrm{H} \beta$ intensity ratios at $v_{\mathrm{r}} \approx 7320 \mathrm{~km} \mathrm{~s}^{-1}$ and $v_{\mathrm{r}} \approx 7200 \mathrm{~km} \mathrm{~s}^{-1}$, the latter value being the peak velocity as derived from the low ionization lines, are $\approx 0.9$ and 3 , re- 

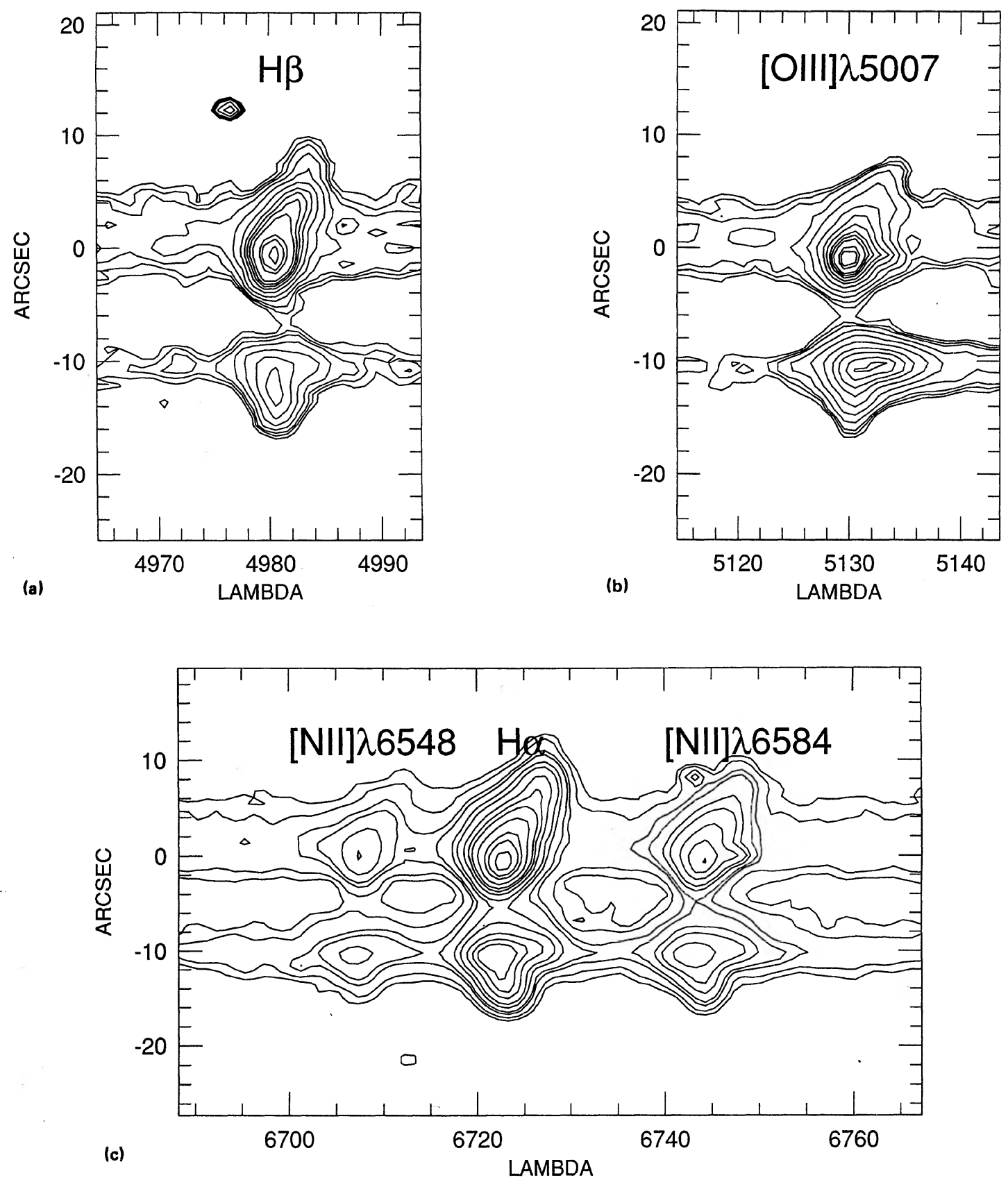

FIG. 6. (a) Spatially extended structure of $\mathrm{H} \beta$, (b) [O III] $\lambda$ 5007, (c) $\mathrm{H} \alpha$ and [N II] $\lambda \lambda$ 6548,6583. Horizontal scale is wavelength in $\AA$, vertical scale is angular distance from $B$ in arcsec.

spectively. This finding shows the coexistence of high and low ionization gas in the blue component.

The ratio [O III] $\lambda 5007 / \mathrm{H} \beta$ measured on the red component decreases monotonically from NGC 7592 A toward NGC $7592 \mathrm{~B}$, and converges to a value $\leqslant 1$ in $\mathrm{B} 2$ where the red component is barely visible, and keeps this value up to $B$. Such decrease moves the point representing the extranuclear red component in the diagnostic diagrams toward the area occupied by the $\mathrm{H}$ II regions, indicating a decrease of the effects produced by the nonthermal nucleus at increasing distances from it. In practice the red component is not present in B2.

The line profiles are narrower in B2 than in A and A2: the mean FWHM is $\approx 150 \mathrm{~km} \mathrm{~s}^{-1}$, and their peak velocity corresponds to the velocity of the blue component. It must be remarked that in $\mathbf{B} 2$, where the effects of ionization by a nonthermal source are absent the peak velocity of the $\mathrm{H} \beta$ and [O III] $\lambda 5007$ lines are in good agreement, further sug- 
gesting that the shift in positions between $\mathrm{H} \beta$ and [O III] in the blue component of $\mathrm{A}$ and $\mathrm{A} 2$ are due to the influence of the Seyfert nucleus.

$N G C 7592 B \equiv M k n 928$. The heliocentric radial velocity of $B$ is $7320 \pm 6 \mathrm{~km} \mathrm{~s}^{-1}$, in good agreement with the value found from the $\mathrm{H} \mathrm{I} 21 \mathrm{~cm}$ emission, $7311 \mathrm{~km} \mathrm{~s}^{-1}$ (Bushouse 1987). Since NGC 7592 B is also the brightest nucleus of the system, we will assume in the following the position and the radial velocity of $B$ as references.

The radial velocities of the [O III] $\lambda \lambda 4959,5007$ lines and of the low ionization lines show no significant discrepancy. Emission line profiles appear to be rather narrow, without differences between Balmer and [O III] $\lambda \lambda$ 4959,5007 lines: the FWHM of $\mathrm{H} \alpha$ and $\mathrm{H} \beta$ are $\approx 200 \mathrm{~km} \mathrm{~s}^{-1}$, while the FWHM of the [O III] $\lambda \lambda 4959,5007$ lines is $\approx 210 \mathrm{~km} \mathrm{~s}^{-1}$. These values are close to those found for the low-ionization lines in NGC $7592 \mathrm{~A}$.

$N G C 7592 C$. NGC 7592 C corresponds to the third condensation revealed by Arkhipova. The $\mathrm{H} \alpha$ luminosity is comparable to that of $\mathrm{B}$, although the continuum is much fainter than that of $B$. the equivalent width of $\mathrm{H} \alpha$ is $\mathrm{W}(\mathrm{H} \alpha) \approx 154 \AA$, two times larger than the value of $\mathrm{B}$. Line profiles are very narrow, and have a mean $F W H M \approx 100$ $\mathrm{km} \mathrm{s}^{-1}$.

\subsection{Emission Line Structure and Velocity Curves}

The spatial emission line structure at P.A. $=100^{\circ}$ is shown in the isophotal maps reported in Figs. 6(a) -6 (c) for the $\mathrm{H} \alpha,[\mathrm{N} \mathrm{II}] \lambda \lambda 6548,6583, \mathrm{H} \beta$ and [O III] $\lambda 5007$ lines. Velocity curves are shown in Fig. 7 (a) (for P.A. $=100^{\circ}$ )
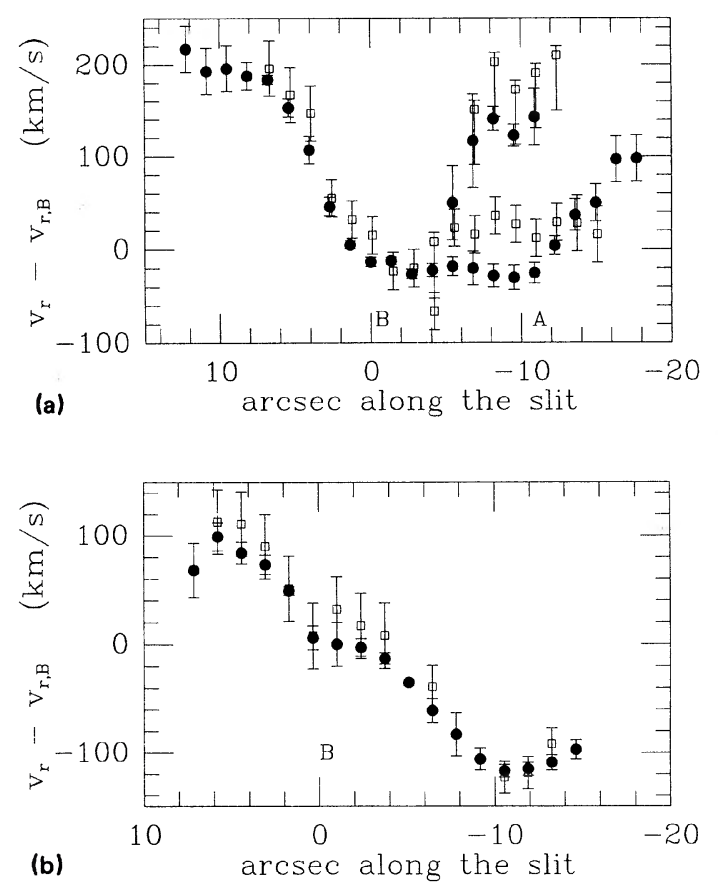

FIG. 7. (a) Radial velocity curves at P.A. $=100^{\circ}$ and (b) at P.A. $=32^{\circ}$ for low ionization (filled circles) and high ionization ( [O III] $\lambda \lambda 4959,5007$ ) lines (open squares). Horizontal scale is angular distance from NGC $7592 \mathrm{~B}$ in arcsec; vertical scale is radial velocity difference from the heliocentric radial velocity of NGC $7592 \mathrm{~B}\left(v_{\mathrm{r}} \approx 7280 \mathrm{~km} \mathrm{~s}^{-1}\right)$. and in Fig. 7(b) (for P.A. $=32^{\circ}$ ), for both the low ionization $(\mathrm{H} \alpha, \mathrm{H} \beta,[\mathrm{N} \mathrm{II}] \lambda \lambda 6548,6583$, [S II] $\lambda \lambda 6716,6731)$, and high ionization lines ([O III] $\lambda \lambda$ 4959,5007). Angular distances and radial velocities are referred to the position and to the heliocentric radial velocity of $B$.

On the eastern side of NGC $7592 \mathrm{~B}$, the radial velocity increases and flattens at around 6 arcsec from B. Between A and $\mathrm{B}$, gas motions appear to be peculiar. The radial velocity curve is separated in two tails, which correspond to the blue and red component described in the previous sections. On the lower tail, the radial velocity curves derived from low ionization and [O III] $\lambda \lambda 4959,5007$ lines differ by about $40-50 \mathrm{~km} \mathrm{~s}^{-1}$ on the western side of $\mathrm{B}$, at locations corresponding to $\mathrm{A}$ and $\mathrm{A} 2$, while they agree within the measurement uncertainties in all other regions. The upper tail is due to gas turning to higher radial velocities (the red component seen on A, A2, and marginally on B2), contributing to both the Balmer and [O III] $\lambda 5007$ emission up to the position of $\mathrm{A}$, and whose $v_{\mathrm{r}}$ increases from $\approx 7300 \mathrm{~km} \mathrm{~s}^{-1}$ to $\approx 7500$ $\mathrm{km} \mathrm{s}^{-1}$. Due to the small separation between the blue and red peak of the [O III] $\lambda \lambda 4959,5007$ lines, values reported for the red component of [O III] $\lambda \lambda 4959,5007$ are upper limits. On the western side of $A$, the velocity curve turns swiftly toward higher radial velocities.

At P.A. $=212^{\circ}$ no systematic differences are found between the velocity curves computed from the low ionization and the [O III] $\lambda \lambda 4959,5007$ lines [Fig. 7(b)]. The velocity curve is approximately linear from $\approx-7$ to $\approx 3$ arcsec from $\mathrm{B}$ and flattens beyond both edges of the linear part.

\section{DISCUSSION}

\subsection{Physical and Kinematical Conditions in NGC 7592 A}

In this section, we will further discuss the ionization mechanism of the blue component detected on NGC 7592 A and A2. In Sec. 3.2.1, we suggested that the location in the diagnostic diagrams of NGC $7592 \mathrm{Ab}$ could be accounted for by the existence of two distinct kinematical components of different ionization degree. Although the ionization mechanism of these components cannot be directly proved, since its presence is revealed only by a slight displacement of the line center of [O III] $\lambda \lambda 4959,5007$ lines, we can give some insight to its ionization mechanism from the following analysis.

If the ionizing source is nonthermal, the gas can be either shock heated or photoionized by a nonthermal source. A contribution from shock-heated gas seems unlikely for explaining the [O III] $\lambda \lambda 4959,5007$ components peaking at $v_{\mathrm{r}} \approx 7330 \mathrm{~km} \mathrm{~s}^{-1}$. If shock-heating plays a role in the ionization, high electron temperature $\left(T_{\mathrm{c}} \sim 10^{5} \mathrm{~K}\right)$ is needed in order to have collisional ionization of $\mathrm{O}^{+}$. In this case, also the strength of lines originating in ions of ionization potential lower than that of $\mathrm{O}^{+}$should be enhanced, and in particular the [N I] $\lambda 5198,[\mathrm{O}$ II] $\lambda \lambda 3726,3729,[\mathrm{~N} \mathrm{II}]$ $\lambda \lambda 6548,6583,[\mathrm{O}$ I] $\lambda 6300$, [S II] $\lambda \lambda 6716,6731$ (Shull \& McKee 1979; Binette et al. 1985). We tested whether the blue components of the strongest low ionization lines ([N II] $\lambda \lambda 6548,6583$, [S II] $\lambda \lambda 6716,6731$ ) showed radial velocity consistent with that of the [O III] $\lambda \lambda 4959,5007$, but we did not reveal any significant difference between the radial velocity of these lines and that of the blue peak of the Balmer lines. 
We reported the intensity ratios [O III] $\lambda 5007 / \mathrm{H} \beta$ and [N II] $\lambda 6583 / \mathrm{H} \alpha$ computed at $v_{\mathrm{r}} \approx 7280 \mathrm{~km} \mathrm{~s}^{-1}\left(\mathrm{Ab}^{-}\right)$ and at $v_{\mathrm{r}} \approx 7330 \mathrm{~km} \mathrm{~s}^{-1}\left(\mathrm{Ab}^{+}\right)$in the diagnostic diagram of Fig. 5(d). The higher ionization degree at $v_{\mathrm{r}} \approx 7330 \mathrm{~km} \mathrm{~s}^{-1}$ definitely locates $\mathrm{Ab}^{+}$in the zone of the nonthermally ionized regions.

Therefore, the [O III] $\lambda \lambda 4959,5007$ line component peaked at $v_{\mathrm{r}} \approx 7330 \mathrm{~km} \mathrm{~s}^{-1}$ is probably being photoionized by an AGN-type nonthermal continuum.

We note in addition that a mixture of gas photoionized by thermal and a nonthermal source can account for the observed line ratios of the blue component. The observed [N II] $\lambda 6548 / \mathrm{H} \alpha$ and [O III] $\lambda 5007 / \mathrm{H} \beta$ ratios can be reproduced assuming that the gas whose ionization source is nonthermal emits a spectrum with $[\mathrm{O}$ III] $\lambda 5007 / \mathrm{H} \beta \sim 5-$ 10 , and [N II] $\lambda 6583 / \mathrm{H} \alpha \sim 1$, that the spectrum of the thermally ionized gas is characterized by line ratios [O III] $\lambda 5007 / \mathrm{H} \beta \sim 1$ and $[\mathrm{N} \mathrm{II}] \lambda 6583 / \mathrm{H} \alpha \sim 0.4$, and that the percentage of contribution of the nonthermally ionized gas to the luminosity of the lines is between $10 \%$ and $30 \%$.

As far as the view suggesting the presence of a mixture of thermally and nonthermally ionized gas is correct, the luminosity in the blue component of the Balmer lines should be essentially due to thermal sources. Assuming that the luminosity of the red component is entirely due to nonthermal sources, the total (both blue and red component) Balmer line luminosity of the gas whose ionization source is nonthermal, is less $(\Sigma 35 \%)$ than that of the gas photoionized by thermal sources.

The centroids of the profiles along the slit of [O III] $\lambda 5007$, of $\mathrm{H} \beta$, and of their nearby continuum are coincident to within the measurement uncertainties $(0.2 \mathrm{arcsec})$ in all the spectra. This implies that the maximum of the [O III] $\lambda 5007$ and $\mathrm{H} \beta$ emission lines originates in a spatially unresolved region coincident with the optical nucleus. Furthermore, we did not find evidence of displacement in the centroid along the slit of the red and blue component. Henceforth, we suggest that thermally and nonthermally ionized gas is simultaneously present in the nucleus and in the nuclear environment of NGC $7592 \mathrm{~W}$. Another point of interest is that the width of the lines emitted by the nonthermally ionized gas are comparable to those of the low ionization, blue line components ( $\leqslant 180 \mathrm{~km} \mathrm{~s}^{-1}$ ). The definition of a local standard of rest from the emission lines of the Seyfert nucleus is not clear: we suggest that the narrow line regions (NLR) of the active nucleus should be associated to the high-ionization gas revealed at $v_{\mathrm{r}} \approx 7330 \mathrm{~km} \mathrm{~s}^{-1}$, the value reported in Table 1 . Although we can only guess that the [O III] $\lambda \lambda 4959,5007$ peak at $v_{\mathrm{r}} \approx 7330 \mathrm{~km} \mathrm{~s}^{-1}$ is due to an additional, nonthermal component, it is difficult to think that the NLR is associated with the red component which is redshifted by $\approx 200 \mathrm{~km} \mathrm{~s}^{-1}$ with respect to the radial velocity of the galaxy, $v_{\mathrm{r}} \approx 7280 \mathrm{~km} \mathrm{~s}^{-1}$ (e.g., Wilson \& Heckman 1985 ). The radial velocity $v_{\mathrm{r}} \approx 7280 \mathrm{~km} \mathrm{~s}^{-1}$ is appropriate as the systemic velocity of the underlying galaxy, since it does probably not suffer from the peculiar motions that could take place in the NLR.

The ambiguity in the definition of the local standard of rest based on the NLR could be responsible for the large differences for the redshift values reported in literature. The $v_{\mathrm{r}}$ value found by us is significantly different from that given by Arkhipova et al. (1981), $\approx 7400 \mathrm{~km} \mathrm{~s}^{-1}$. The agreement is not satisfactory also with the value $v_{\mathrm{r}}=7255 \mathrm{~km} \mathrm{~s}^{-1}$ found by Dahari (1985). The lower resolution ( $\approx 13 \AA$ ) of Dahari's spectra did not allow to separate the blue and red line components, thus probably shifting the line center toward the stronger, blue, low-ionization component.

\subsection{Geometry of the Encounter and Dynamical Masses}

The detection of the plume toward west revealed on NGC $7592 \mathrm{~S}$, and the distortion in the arc of condensations in the south of NGC 7592 B suggest that NGC $7592 \mathrm{~S}$ is likely to be a third galaxy physically interacting with NGC $7592 \mathrm{~W}$ and E.

In order to gain further clues to the geometry of the system, we need to actually explain the two extensions seen on NGC $7592 \mathrm{~W}$ as origins of two spiral arms. If spiral arms are trailing, NGC $7592 \mathrm{~W}$ should rotate clockwise, and since in the western part of the galaxy the gas is receding the observer [see Fig. 7(a) ], it follows that the wing is in foreground with respect to the galactic bulge. In this case, NGC $7592 \mathrm{~W}$ can be described as a rather unperturbed galaxy, probably of morphological type Sa, located behind NGC $7592 \mathrm{E}$ and whose southern spiral arm is hidden by the galactic body of NGC 7592 E. Identification of a spiral structure in NGC $7592 \mathrm{E}$ is hampered by the perturbed morphology of this galaxy. It is not possible to state with reliability whether the arc of condensations described in Sec. 3.1 can be assimilated to a spiral arm. Another possibility would be to consider it as the remnant of a ringed structure. However, if the interpretation of the morphology of NGC $7592 \mathrm{~W}$ is correct, the southeastern part of NGC $7592 \mathrm{E}$ should be closer to the observer than the northwestern edge. In this case, the radial velocity curve at P.A. $=100^{\circ}$ allows to state that gas in the contact region between NGC $7592 \mathrm{E}$ and W rotates in opposite directions.

We have pointed out earlier that it is difficult to unambiguously define a standard of rest for NGC $7592 \mathrm{~A}$, but that the radial velocity deduced from the blue component of the low-ionization lines, $v_{\mathrm{r}} \approx 7280 \mathrm{~km} \mathrm{~s}^{-1}$ is probably related to the underlying galaxy. The radial velocity of the nucleus of NGC 7592 B slightly exceeds that of NGC 7592 A, by $\Delta v_{\mathrm{r}} \approx 40 \mathrm{~km} \mathrm{~s}^{-1}$. Henceforth, the orbital motion of NGC $7592 \mathrm{~W}$ appears to be in the same sense of the rotational motion of NGC $7592 \mathrm{E}$, suggesting that a prograde encounter is taking place. A prograde encounter in which the disks of both galaxies rotate in the same direction in their contact region is possible if the northern wing of NGC $7592 \mathrm{~W}$ is located beyond the bulge of NGC $7592 \mathrm{~W}$. This could be the case if the wing were produced by tidal effects, but it seems to be rather unlikely: Toomre \& Toomre (1972) pointed out that tidal arms and tails are usually very elongated, narrow features. The northern wing of NGC $7592 \mathrm{~W}$ is on the contrary very broad, even as a spiral arm, unless the arm suffered a strong perturbation in the past. In our case, for instance, it is possible that NGC $7592 \mathrm{~S}$ passed in the vicinity of the arm, acting as a perturber.

The inclination of NGC $7592 \mathrm{E}$ can be computed from the ellipticity of the arc of condensation. We estimated $i \approx 44^{\circ}$. The position angle of the nodal line $\phi$ can be estimated with good accuracy by the comparison of the radial velocities observed at P.A. $=32^{\circ}$ and $100^{\circ}$. Following the procedure of Burbidge et al. (1960), we obtained $\phi \approx 91^{\circ}$. The rotational velocity $v_{\text {rot }}$ is thus $\approx 270 \mathrm{~km} \mathrm{~s}^{-1}$ at a deprojected distance from $\mathrm{B} d \approx 3.0 h^{-1} \mathrm{kpc}$. The value of the rotational velocity is typical of galaxies of morphological type $\mathrm{Sa} / \mathrm{Sab}$ (e.g., 
Rubin et al. 1984). We estimated the mass contained within this radius $\mathscr{M}=G^{-1} R v_{\text {rot }}^{2}(R) \approx 5.0 \times 10^{10} \mathscr{M}_{\odot}$. It is very difficult to get a reliable estimate of the inclination of NGC $7592 \mathrm{~W}$, but the general appearance suggests that it is seen rather face-on. Conversion to true rotational velocity (and hence mass estimate of NGC $7592 \mathrm{~W}$ using the dynamical method) for the radial velocities observed on the western side of $\mathrm{A}$ is hampered by the large uncertainty in the inclination estimate, and by the lack of a certain criterion for evaluating the position angle of the nodal line $\phi$. However, we note that on the western side of $\mathrm{A}$, radial velocity increases up to $v_{\mathrm{r}} \approx 150 \mathrm{~km} \mathrm{~s}^{-1}$, suggesting that rotational velocities could be as high as or even higher than for NGC 7592 E. The small size and the intrinsic faintness of the stellar continuum of NGC $7592 \mathrm{~S}$ indicates that this component has probably a mass much lower than that of NGC $7592 \mathrm{E}$ and $\mathrm{W}$. The large EW of $\mathrm{H} \alpha$ and the infrared colors typical of a giant $\mathrm{H}$ II complex (Bushouse \& Werner 1990) question even the nature of NGC $7592 \mathrm{~S}$ as a galactic body. An estimate of the mass of NGC $7592 \mathrm{~W}$ is anyway possible analyzing the gas motions in the contact region of NGC $7592 \mathrm{E}$ and W.

\subsubsection{Between NGC 7592 A and B: infall or outflow?}

We have shown that the spatially extended emission line gas in the region between the nuclei of NGC $7592 \mathrm{E}$ and W, and in the nucleus of NGC $7592 \mathrm{~A}$, is made up of two principal components. The blue component is probably emitted in the nuclear and disk star forming regions of the system, with a contribution to the ionization from a nonthermal source. The red component is redshifted with respect to the systemic velocity of NGC $7592 \mathrm{~W}$ and is due to high-excitation gas, photoionized by a nonthermal source (see Sec. 3.2). It appears that the motions of the red component cannot be readily explained in terms of rotation. The geometry of the system NGC 7592 could indicate that the gas receding the observer, emitting the red component, is moving toward NGC 7592 $\mathrm{W}$, located behind NGC $7592 \mathrm{E}$. An alternative hypothesis is that the gas is outflowing from NGC $7592 \mathrm{~A}$. In this case, we should be able to see gas moving on the far side of NGC 7592 $\mathrm{W}$, regardless of the geometry of the system. One has to explain why the gas seems to converge to the radial velocity of the low ionization gas, in correspondence of $\mathrm{B}$, and to find a suitable mechanism for matter ejection up to a projected distance of $\sim 5 h^{-1} \mathrm{kpc}$. Both circumstances seem to be rather unlikely. In addition, the blue component suffers a stronger reddening $[\mathrm{E}(B-V) \approx 0.24]$ than the red one (which is nearly unreddened). This further indicates that the gas emitting the red component is probably located in foreground with respect to the gas emitting the blue component. Thus we believe that the most reasonable assumption is that the gas emitting the red component is infalling toward NGC 7592 A.

Under this assumption, we can compute the mass of the attracting galaxy. The maximum radial velocity with respect to the Seyfert nucleus is $v_{\mathrm{r}} \approx 200 \mathrm{~km} \mathrm{~s}^{-1}$, and it is reached at a (projected) distance of $\approx 500$ pc. Applying a correction factor for the projected velocity $\sin i \approx \sqrt{2} / 2$, we obtain a lower limit to the mass of NGC $7592 \mathrm{~W}, \mathscr{M} \approx 4.8 \times 10^{9} \mathscr{M}_{\odot}$.

\subsection{Star Formation}

A comparison of the $\mathrm{H} \alpha$ luminosity of the emitting regions of NGC 7592 with the $\mathrm{H} \alpha$ luminosity function for starburst galactic nuclei published by Kennicutt et al.
(1989) shows that the $\mathrm{H} \alpha$ luminosity of $\mathrm{B}$ and $\mathrm{C}$ are typical of this class of objects and that they would be extremely high for disk $\mathrm{H}$ il regions. The $\mathrm{H} \alpha$ luminosity of $\mathrm{Ab}$, although lower than that of $\mathrm{B}$ by a factor $\sim 2$, is still common among starburst nuclei. The $\mathrm{H} \alpha$ luminosity of the extranuclear regions (Table 2) is also located on the high-luminosity tail of the $\mathrm{H} \alpha$ luminosity function for disk $\mathrm{H}$ II regions of Kennicutt et al. (1989).

The star formation rate (SFR) can be computed from the reddening corrected $\mathrm{H} \alpha$ luminosity, with a suitable choice of the initial mass function (IMF). Adopting an extended Miller-Scalo IMF, where $\Psi(\mathscr{M}) \propto \mathscr{M}^{-1.4}$ for $0.1 \mathscr{M}_{\odot}<\mathscr{M}<1 \mathscr{M}_{\odot} \quad$ and $\quad \Psi(\mathscr{M}) \propto \mathscr{M}^{-2.5} \quad$ for $1 \mathscr{M}_{\odot}<\mathscr{M}<100 \mathscr{M}_{\odot}$, and following Kennicutt (1983) we obtain the results reported in Table 3 for the high mass $\left(\mathscr{M}>10 \mathscr{M}_{\odot}\right)$ SFR, and for the total SFR. For NGC 7592 A, we compute the SFR taking the thermal contribution of the $\mathrm{H} \alpha$ luminosity, which corresponds, as an upper limit, to the $\mathrm{H} \alpha$ luminosity of $\mathrm{Ab}$, about $3 / 4$ of the total $\mathrm{H} \alpha$ luminosity. SFR per unit of projected area (SFR/area) has the largest values in NGC $7592 \mathrm{E}$ in correspondence of the nucleus $\mathrm{B}$, according to the finding that the SFR/area is usually larger in the nuclear regions than in the circumnuclear and disk regions (Bushouse 1987). It is interesting to note that this is not true for the thermal contribution in NGC $7592 \mathrm{~A}$, whose lower limit to the SFR/area is comparable to that of $\mathrm{A} 1$, which is probably made up by unresolved disk $\mathrm{H}$ II regions. Far-infrared fluxes (between 25 and $100 \mu \mathrm{m}$ ) of the whole system allow an independent estimate of the SFR.

NGC 7592 is known as an IRAS source, whose $\mathrm{SFR} \approx 33 h^{-2} \mathscr{M}_{\odot} \mathrm{yr}^{-1}$ appears to be very high also for interacting galaxies (Bushouse 1987). This value is larger than the values found employing the reddening corrected emission line luminosity ( $\approx 7.6 h^{-2} \mathscr{M}_{\odot} \mathrm{yr}^{-1}$ ). This discrepancy is quite likely due to the fact that our values refer only to the area covered by the slit of the spectrograph, while the IRAS fluxes are to be referred to the whole system, whose angular extension is much less than the slit aperture of IRAS. However, the $\mathrm{H} \alpha$ fluxes reported by Bushouse (1987) and obtained through $\mathrm{H} \alpha$ narrow band imaging yield a total SFR for NGC $7592 \approx 2.6 h^{-2} \mathscr{M}_{\odot} \mathrm{yr}^{-1}$. Applying an average reddening correction $[\mathrm{E}(B-V) \approx 0.48$, the average color excess for the emitting regions isolated in our spectra], the SFR becomes $\approx 7.6 h^{-2} \mathscr{M}_{\odot} \mathrm{yr}^{-1}$, the same value derived from our observations. This implies that our observations cover all the regions where the SFR/area has high values, and/or where the internal reddening is low.

It is interesting to compute the age over which the starburst phase can be sustained at the present SFR, e.g., the time needed to completely exhaust the gas reservoir of the galaxy. The total $\mathrm{H}_{\mathrm{I}}$ mass of NGC 7592 is $\mathscr{M}_{\mathrm{H}_{\mathrm{I}}} \approx 6.85 \times 10^{9} h^{-2} \mathscr{M}_{\odot}$ (Bushouse 1987). The total gas mass was obtained by multiplying the total $\mathrm{H}$ I mass by a factor 2.0 to account for Helium and molecular gas and by 1.25 to account for recycled gas, following Larson et al. (1980). The value of the depletion time is either $\approx 5 \times 10^{8} \mathrm{yr}$ or $2 \times 10^{9} \mathrm{yr}$ assuming that the SFR for the whole system is either $\approx 33 h^{-2} \mathscr{M}_{\odot} \mathrm{yr}^{-1}$ or $\approx 7.6 h^{-2} \mathscr{M}_{\odot} \mathrm{yr}^{-1}$. The first value (only a few tens the lifetime of O-B stars, $t_{\mathrm{OB}} \sim 2 \times 10^{7} \mathrm{yr}$ ), which is probably more appropriate since the IRAS fluxes are not affected by internal extinction, underlines the temporary character of the star formation events occurring in NGC 7592. 


\subsection{Chemical Composition}

Emission line fluxes were corrected for internal reddening according to the color excess $\mathrm{E}(\boldsymbol{B}-\boldsymbol{V})$ tabulated in Table 3 . We then employed the extragalactic $\mathrm{H}$ il regions abundance sequence build by Dopita \& Evans (1986), and the method recommended by these authors for computing the abundance of oxygen, nitrogen, and sulfur.

Computation of the element abundances has been limited to the regions $\mathrm{B}, \mathrm{B} 3, \mathrm{~B} 4, \mathrm{C}$, for which reliable [O II] $\lambda \lambda 3726,3729$ fluxes are available. The method of Dopita \& Evans (1986) could not be applied in region A, for which the contribution of the nonthermally ionized gas can be separated in all the relevant lines but not for the [O II] $\lambda \lambda$ 3726,3729 doublet. Results are reported in Table 4. The oxygen abundance of the B, B3, and B4 regions are the same to within the uncertainties, and approximately $15 \%$ larger than the solar values. Conversely, the oxygen abundance for the $\mathrm{C}$ region is significantly lower, namely $\approx 10 \%$ below the solar values. Nitrogen and sulfur abundances relative to oxygen are not different from the solar value, within the uncertainties.

\subsection{The Nature of the Semibroad Component of [O III] $\lambda \lambda 4959,5007$}

Recent work outlines that red asymmetries are indeed present in the [O III] $\lambda \lambda 4959,5007$ lines, although at present the percentage of known objects showing a blue asymmetry is higher (Busko \& Steiner 1989). This view is clearly different from that of a few years ago, when it was commonly believed that red asymmetries had never been observed (e.g., Wilson \& Heckman 1985). The strong red asymmetry found in the [O III] $\lambda \lambda 4959,5007$ lines of NGC $7592 \mathrm{~A}$ is not a unique feature, although it is not common. The asymmetry index, defined following Whittle (1985), is $\mathbf{A I}=-0.3$, and would place the profile at the limit of the asymmetry index distribution for the [O III] lines in the sample of Busko \& Steiner (1989). There is one object (at least) showing an [O III] line profile very similar to that observed on NGC $7592 \mathrm{~W}$, namely NGC 4388 (Iye \& Ulrich 1986). In the previous sections we have outlined the evidence suggesting that the red component of NGC $7592 \mathrm{~W}$ is due to gas ultimately infalling toward NGC $7592 \mathrm{~A}$. Since the radial velocity and the ionization degree (e.g., the $[\mathrm{O}$ III $] \lambda 5007 / \mathrm{H} \beta$ ratio) appear to increase steadily approaching the Seyfert nucleus, it is plausible that the semibroad component could be also due to gas in-falling toward the central core of the galaxy. Although we can not prove this suggestion, we can

TABLE 4. Element abundances.

\begin{tabular}{cccc}
\hline \hline & $12+\log (\mathrm{O} / \mathrm{H})$ & $\log (\mathrm{N} / \mathrm{O})$ & $\log (\mathrm{S} / \mathrm{O})$ \\
\hline B & & & \\
B3 & 8.86 & -1.16 & -1.58 \\
B4 & 8.85 & -1.20 & -1.52 \\
C & 8.87 & -1.15 & -1.60 \\
Adopted Solar & 8.75 & -1.15 & -1.61 \\
\hline
\end{tabular}

show that the ionization source can be provided by the Seyfert nucleus itself, and that the increase of the ionization degree is consistent with the gas experiencing a stronger photoionization because of its location closer to the Seyfert nucleus.

Emission of the semibroad component is not spatially resolved, so that it should arise in a region contained within approximately $\pm 180 \mathrm{pc}$ from the very center of NGC 7592 W. Moreover, we are able to estimate a lower limit to the ratio [O III] $\lambda 5007 / \mathrm{H} \beta \gtrsim 5$ taking the intensity ratio at $\Delta v_{\mathrm{r}} \approx 450 \mathrm{~km} \mathrm{~s}^{-1}$ from the blue peak of the [O III] $\lambda 5007$ line, where the emission in [O III] $\lambda 5007$ begins to be due only to the semibroad component. The analysis of Sec. 3.2 suggests that the heating mechanism of the gas emitting the semibroad component is provided by a nonthermal source. In the following, we further suggest that the emitting gas is able to see the ionizing continuum from the Seyfert nucleus, and that the ionization degree should be higher in comparison to that of the gas emitting the red component.

Under the assumption of photoionization, the ratio [O III ] $\lambda 5007 / \mathrm{H} \beta$ is sensitive, for a fixed metallicity, mainly to the ionization parameter $\Gamma=Q(H) / 4 \pi R^{2} n_{\mathrm{e}} c$, where $R$ is the distance from the photoionizing source, and $Q(H)$ is the number of ionizing photons. In order to compute $\Gamma$, $Q(H)$ can be estimated from the reddening corrected value of the $\mathrm{H} \alpha$ luminosity, taking the nonthermal contribution ( $\approx 25 \%$ of the total line luminosity). It results that $Q(H)_{\mathrm{A}} \sim 1.0 \times 10^{52} h^{-2}$ photon $\mathrm{s}^{-1}$. Then, a lower limit to $\Gamma$ is $\approx 1.5 \times 10^{-4}$, if the gas is located at $R \approx 200$ pc from the central source. Ferland \& Netzer (1983) computed the dependence upon $\Gamma$ of several line ratios. According to their diagrams, a ratio $[\mathrm{O} \mathrm{III}] \lambda 5007 / \mathrm{H} \beta \approx 5$ implies a value of the ionization parameter of $\approx 3.5 \times 10^{-4}$, in the case of solar metallicity. This value of $\Gamma$ is comparable with that computed directly, suggesting that the nucleus can be the ionization source for the semibroad line region. The FWZI of the semibroad component allows the computation of the central mass, $\mathscr{M}_{\mathrm{c}}=R_{\min } v_{\max }^{2} / 2 G$, where $R_{\min }$ is the minimum distance reached from the Seyfert nucleus. Since $v_{\max } \approx 1200$ $\mathrm{km} \mathrm{s}^{-1}, \mathscr{M}_{\mathrm{c}} \approx 1.74 \times 10^{8} R_{\text {min }}(\mathrm{pc}) \mathscr{M}_{\odot}$. Since the ionization parameter could be as high as $\sim 10^{-3}-10^{-2}$, but the density cannot greatly exceed the critical density for the [O III] $\lambda \lambda 4959,5007$ lines, we can assume that $\Gamma n_{\mathrm{e}} \sim 10^{4.5}-10^{3.5}$. Hence, $R_{\min }=\left[Q(H) / 4 \pi c\left(\Gamma n_{\mathrm{e}}\right)\right]^{1 / 2}$ $\sim 0.37-1.2$ pc. The central mass is thus $\mathscr{M}_{\mathrm{c}} \sim 6.5 \times 10^{7}-2.1$ $\times 10^{8} \mathscr{M}_{\odot}$, a reasonable value for Seyfert galaxies.

\section{CONCLUDING REMARKS}

We find evidence in NGC 7592 A of a nuclear starburst occurring simultaneously with Seyfert type activity. This is not a unique case among Seyfert galaxies: NGC 7469, studied by Wilson et al. (1986), shows also a nuclear starburst in addition to Seyfert characteristics. In this and in other known cases (NGC 1365, NGC 1068, NGC 7582, Mkn 509, Wilson et al. 1986) the luminosity of the Seyfert component usually overswamps the luminosity of the nuclear starburst. An interesting feature for NGC 7592 is that the emission line luminosity of the nuclear starburst in A is apparently much larger than that due to the Seyfert type activity. It is moreover interesting to note that the high ionization gas responsible for the Seyfert character of the line spectrum shows line widths comparable to those of the low ionization $\mathrm{H}$ II region. This may suggest that the Seyfert activity could arise from 
the same conditions from which the starburst phenomenon is formed. We note moreover that the star formation rate per unit area in the Seyfert nucleus is less than that deduced for A1, unlike the case of truly starbursting and interacting system, for which the SFR/area is strongly peaked near to the center of the galaxy (Bushouse 1987), as is the case of NGC $7592 \mathrm{E}$.

Interaction is able to trigger star formation in a more $d i$ rect way than nuclear activity, as it seems to be the case from several evidences partly reviewed in the introduction of this work. The insurgence of Seyfert activity in interacting systems could be delayed by the time necessary for the gas to accrete the core, while starburst could be produced in earlier stages of interaction. Assuming an attracting mass $\sim 10^{11}$ $\mathscr{M}_{\odot}$ (a reasonable value for NGC $7592 \mathrm{~W}$ ), and that gas is located initially at $\approx 3.5 \mathrm{kpc}$ from $\mathrm{A}$, a lower limit to the time needed for the gas to reach the Seyfert nucleus from the vicinity of $B$ is given by the free-fall time (from rest), $t_{\mathrm{ff}}=V(3 \pi / 32 G \rho) \approx 1 \times 10^{8} \mathrm{yr}$. This is more than the lifetime of a generation of O-B stars, $t_{\mathrm{OB}} \sim 2 \times 10^{7} \mathrm{yr}$. We suggest that transfer of matter is taking place between NGC $7592 \mathrm{E}$ and $\mathrm{W}$, in the sense that gas is moving toward the Seyfert galaxy, and it is (possibly) accreting the central core of the nucleus. Our analysis has shown that the gas has already reached the Seyfert nucleus, while the starburst is still occurring. The slightly over solar chemical abundances obtained for NGC 7592 E may indicate that we are observing a generation of O-B stars later than the first one. The dynamical properties of this extremely interesting system could be responsible for the ongoing exchange of matter and for the location and intensity of the star formation events. If NGC $7592 \mathrm{~S}$ is indeed a third galactic body, which most probably experienced a star formation history different from NGC $7592 \mathrm{E}$ and $\mathrm{W}$, since the $\mathrm{O} / \mathrm{H}$ ratio is less than solar and significantly different from that of NGC $7592 \mathrm{E}$.

The prograde encounter between NGC $7592 \mathrm{E}$ and W is quite likely responsible for most morphological peculiarities, and for the transfer of matter as suggested by Toomre \& Toomre (1972). If a third galaxy, NGC $7592 \mathrm{~S}$ of mass much smaller than that of NGC $7592 \mathrm{E}$ and W, is indeed present in the system, an analogy with the restricted threebody problem could be drawn, since the three galaxies would be nearly equidistant from each other. This could be an alternative good premise for a complete theoretical modeling, able to explain the morphological peculiarities and the ongoing transfer of matter.

\section{REFERENCES}

Adams, T. F. 1977, ApJS, 33, 19

Arkhipova, V. P., Afanas'ev, V. L., Dostal', V. A., Zasov, A. V., Karachentsev, I. D., \& Noskova, R. I. 1979, SvA, 25, 277

Baldwin, J. A., Phillips, M. M., \& Terlevich, R. 1981, PASP, 93, 5

Balzano, V. A. 1983, ApJ, 268, 602

Binette, L., Dopita, M. A., \& Tuohy, I. R. 1985, ApJ, 297, 476

Burbidge, G. R., Burbidge, E. M., \& Prendergast, K. H. 1960, ApJ, 131, 282

Burstein, D., \& Heyles, C. 1984, ApJS, 54, 33

Bushouse, H. A. 1987, ApJ, 320, 49

Bushouse, H. A., \& Werner, M. W. 1990, ApJ, 359, 72

Busko, I. C., \& Steiner, J. E. 1989, MNRAS, 238, 1479

Byrd, G. G., Valtonen, M. J., Sundelius, B., \& Valtaoja, L. 1986, A\&A, 166, 75

Dahari, O. 1984, AJ, 89, 966

Dahari, O. 1985, ApJS, 57, 643

Dahari, O., \& de Robertis, M. M. 1988a, ApJS, 67, 249

Dahari, O., \& de Robertis, M. M. 1988b, ApJ, 331, 727

De Robertis, M. M. 1985, AJ, 90, 998

De Robertis, M. M., \& Shaw, R. A. 1988, ApJ, 329, 629

de Vaucouleurs, G., \& de Vaucouleurs, A. 1964, 2nd Reference Catalogue of Bright Galaxies (The University of Texas Press, Austin)

Dopita M. A., \& Evans I. N. 1986, ApJ, 307, 431

Feldman, F. R., Weedman, D. W., Balzano, V. A., \& Ramsey, L. W. 1982, ApJ, 256, 427

Ferland, G. J., \& Netzer, H. 1983, ApJ, 264, 105

Fuentes-Williams Stocke, J. T. 1988, AJ, 96, 1235

Gunn, J. 1979, in Active Galactic Nuclei, edited by C. Hazard and S. Mitton (Cambridge University Press, Cambridge), p. 213

Heckman, T. M. 1982, ApJ, 268, 602

Hummel, E., Pedlar, A., van der Hulst, J. M., \& Davies, R. D. 1985, A\&AS, 60, 293

Iye, M., \& Ulrich, M. H. 1986, Ap\&SS, 118, 523

Keel, W. C., Kennicutt, R. C., van der Hulst, J. M., \& Hummel, E. 1985, AJ, 90, 708

Kennicutt, Jr., R. C. 1983, ApJ, 272, 54

Kennicutt, Jr., R. C., Keel, W. C., \& Blaha, C. A. 1989, AJ, 97, 1022
Kennicutt, Jr., R. C., \& Keel W. C. 1984, ApJ, 279, L5

Larson, R. B., Tinsley, B. M., \& Caldwell, C. N. 1980, ApJ, 237, 692

Lawrence, A., Rowan-Robinson, M., Leech, K., Jones, D. M. P., \& Wall, J. MNRAS, 249, 329

Lonsdale, C. J., Persson, S. E., \& Matthews, K. 1984, ApJ, 287, 95

Markarian, B. E., Lipovetskii, V. A., \& Stepanyan D. A. 1977, Ap, 13, 116

Miley, G. K., Neugebauer, G., \& Soifer, T. B. 1985, ApJ, 293, L11

Miller, J. S., \& Mathews, W. G. 1972, ApJ, 172, 593

Norman, C., \& Scoville, N. 1988, ApJ, 332, 124

Pritchet, C., \& Monacki, S. 1982, PASP, 84, 733

Rafanelli, P. 1989, in Active Galactic Nuclei, IAU Symposium No. 134, edited by D. E. Osterbrock and J. S. Miller (Reidel, Dordrecht), p. 478

Rodriguez-Espinoza, J. M.., Rudy, R. J., \& Jones, B. 1987, ApJ, 312, 555

Rubin, V. C., Burstein, D., Ford, Jr., W. K., \& Thonnard, N. 1984, ApJ, 289,81

Shull, T. M., \& McKee, C. F. 1979, ApJ, 178, 623

Simkin, S. M., Su, H. J., \& Schwarz, M. P. 1980, ApJ, 237, 404

Stocke, J. T. 1978, AJ, 83, 348

Stockton, A. 1982, ApJ, 257, 33

Stone, R. P. S. 1977, ApJ, 218, 767

Sulentic J. W. 1976, ApJ, 213, 327

Terndrup, D., Lauer, T. R., \& Stover, R. J. 1984, Lick Obs. Tech. Rep., No. 33

Toomre, A., \& Toomre, J. 1972, ApJ, 178, 623

Veilleux, S., \& Osterbrock, D. E. 1987, ApJS, 63, 295

Vorontsov-Veliaminov, B. A. 1979, A\&AS, 28, 1

Weedman, D. W. 1983, ApJ, 266, 479

Weedman, D. W. 1989, in Star Formation in Galaxies, NASA Conf. Publ. No. 2466 (NASA, Washington, DC), p. 351

Whittle, M. 1985, MNRAS, 213, 1

Wilson, A. S. 1988, A\&A, 206, 401

Wilson, A. S., Baldwin, J. A., Sun, S. D., \& Wright, A. E. 1986, ApJ, 310, 121

Wilson, A. S., \& Heckman, T. M. 1985, in Astrophysics of Active Galaxies and Quasi Stellar Objects, edited by J. S. Miller (University Science Books, Mill Valley), p. 39 


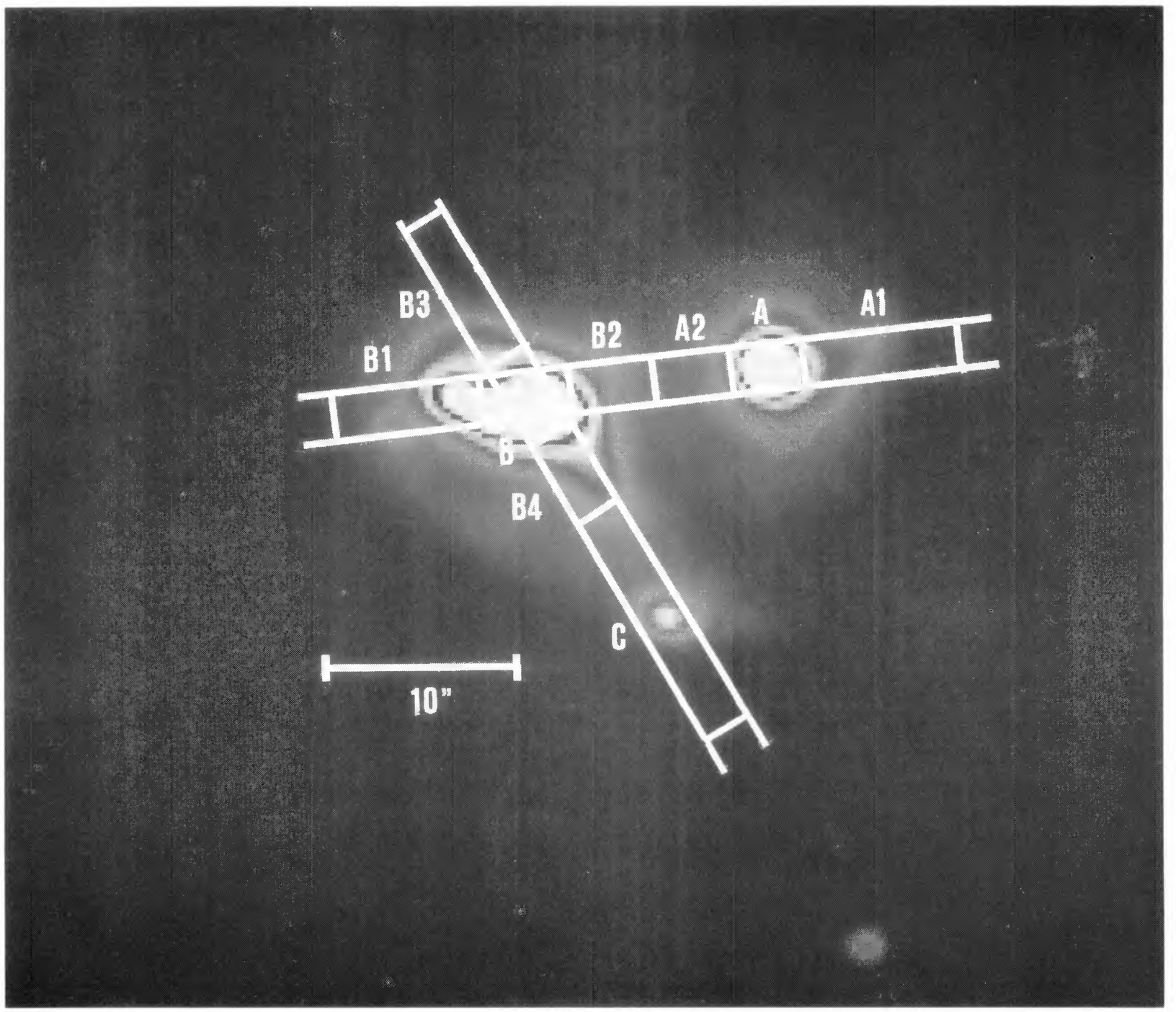

FIG. 1. $R$-band image of NGC 7592. North is to the top, east is to the left. Emission regions are marked as defined in Table 1 .

P. Rafanelli and P. Marziani (see page 745) 